\title{
Toward the reconstruction of Proto-Algonquian-Wakashan. Part 3: The Algonquian-Wakashan 110-item wordlist
}

\begin{abstract}
In the third part of my complex study of the historical relations between several language families of North America and the Nivkh language in the Far East, I present an annotated demonstration of the comparative data that was used in the lexicostatistical calculations to determine the branching and approximate glottochronological dating of Proto-AlgonquianWakashan and its offspring; because of volume considerations, this data could not be included in the previous two parts of the present work and has to be presented autonomously. Additionally, several new Proto-Algonquian-Wakashan and Proto-Nivkh-Algonquian roots have been set up in this part of study. Lexicostatistical calculations have been conducted for the following languages: the reconstructed Proto-North Wakashan (approximately dated to ca. $800 \mathrm{AD}$ ) and modern or historically attested variants of Nootka (Nuuchahnulth), Amur Nivkh, Sakhalin Nivkh, Western Abenaki, Miami-Peoria, Fort Severn Cree, Wiyot, and Yurok.

Keywords: Algonquian-Wakashan languages, Nivkh-Algonquian languages, Algic languages, Wakashan languages, Chimakuan-Wakashan languages, Nivkh language, historical phonology, comparative dictionary, lexicostatistics.
\end{abstract}

The classification and preliminary glottochronological dating of Algonquian-Wakashan currently remain the same as presented in Nikolaev 2015a, Fig. $1^{1}$. That scheme was generated based on the lexicostatistical analysis of 110-item basic word lists ${ }^{2}$ for one reconstructed (Proto-Northern Wakashan, ca. 800 A.D.) and several modern Algonquian-Wakashan languages, performed with the aid of StarLing software ${ }^{3}$. Etymologies for the overwhelming majority of the 110-item wordlist entries have been established based on the list of regular sound correspondences as suggested in Nikolaev 2015a, §3; whenever those correspondences are generally satisfied, we surmise that the respective items represent results of genetic divergence, rather than diffusion and borrowing occurring already after the disintegration of the original Proto-Algonquian-Wakashan.

The third publication in this ongoing series is presented in the form of an annotated demonstration of the comparative data that was used in lexicostatistical calculations; since this presentation sometimes demands detailed comments on various aspects of historical phonology that take up too much space, it could not have been included in the previous two publications on the subject.

Certain improvements to the ongoing work on PAW and PNA reconstruction have been produced during the preparation of the present work; consequently, a few differences from Nikolaev 2015b are to be encountered. These are consistently marked in the main body of the

${ }^{1}$ None of these glottochronological dates should be accepted as incontestable facts; undoubtedly, certain details will be liable to change as the material of all the other Algonquian-Wakashan subgroups (primarily Quileute and perhaps also Kutenai) is added to the comparison. Current datings should be understood as reflecting a highly approximate temporal scale for language divergence.

2 The standard 100-item Swadesh wordlist with 10 additional words for the purpose of more accurate classification and dating of the cognate languages.

3 StarLing for Windows v. 2.5 .3 (computerized system for multilingual database processing; copyright 1985-2005 by Sergei Starostin; http://starling.rinet.ru).

Journal of Language Relationship • Вопросы языкового родства • 15/4 (2017) • Pp. 250-278 • ( The authors, 2017 
work with the abbreviation “cf.", e. g.: PAW *m'a:whV $\approx$ *ham'wV 'to eat, bite' (cf. Nikolaev 2015b, \#234), reflecting the fact that in Nikolaev 2015b this root was reconstructed as ${ }^{*} m^{\prime} a: h V \approx{ }^{*} h a m$ ' $V$.

The following new PAW and PNA rooots have been added: PAW * $k$ ' $: x k V(\sim k$ ' $g$ ) 'all, every'; PAW *w'adV $\chi E$ 'sky, cloud'; PAW *mi:- 'food, fish; to eat'; PAW * $q^{w} o: K t V$ 'full'; PAW * $y a: k V(\sim g)$

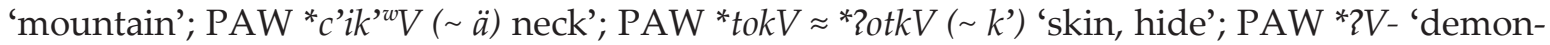
strative stem'; PAW * $q V(\sim \mathrm{G})$ 'interrogative stem'; PAW * $2 A \eta$ 'interrogative stem'; PAW ${ }^{*} h V_{3} V$ $\approx{ }^{*} 2 V h_{3} V$ 'tail (of quadruped)'; PNA *tu(:) $)_{6} w V(\sim 0)$ 'to burn (tr.)'. Conversely, the roots PNA ${ }^{*} x^{w} a \approx{ }^{*} h a x^{w} V$ 'name'(Nikolaev 2015b, \#389), PAW * $p E: s ̌ V \approx{ }^{*} \imath E: p s ̌ V$ 'one' (Nikolaev 2015b, \#281) and PNA *tOyViwV'to burn (tr.)' (Nikolaev 2015b, \#345) have been eliminated as non-existent.

In the main body of the wordlists, language forms and their meanings that are relevant for lexicostatistical calculations, are given in bold print. They include: (a) reconstructed forms for Proto-North Wakashan (glottochronologically dated to ca. 800 AD) ${ }^{4}$; (b) Southern Wakashan Nootka (Nuuchahnulth); (c) Nivkh - Amur and Sakhalin; (d) Algonquian - Western Abenaki, Miami-Peoria, Fort Severn Cree; (e) other Algic languages - Wiyot and Yurok. All data on languages from groups (b-e) have been taken from sources recorded over the 19th and 20th centuries.

All the data are given in etymological order: etymologically different roots are listed under separate numbers in round brackets - (1), (2), etc. The symbol • is used to separate language families, whereas $\boldsymbol{\Delta}$ is used to mark different root variants within the same family. The equation symbol $(=)$ indicates that the root allomorph is used exclusively with possessive prefixes; the hyphen (-) is used to separate any other morphemes (regardless of their degree of productivity in the given language).

In protoforms the tilde symbol $(\sim)$ denotes alternately possible variants of reconstruction, rather than an actual alternation in the protolanguage. If reconstruction of two (rarely three or more) protophonemes in the same position is possible, alternate variants are given in round brackets. Many PAW roots are represented by "inversed" allomorphs ${ }^{*} C V C V$ and *2VCCV (more rarely, ${ }^{*} h V C C V$ ). Where present, reconstructed allomorphs of this type are divided by double tilde $(\approx)$.

Latest results of lexicostatistical calculations between all these languages, reflecting percentages of lexical cognacy, are adduced below in Table 1 (for the revised 110-item wordlist) and in Table 2 (for the revised 50-item wordlist).

Table 1. Percentage of lexical cognacy between Algonquian-Wakashan languages (110-item wordlist)

\begin{tabular}{l|c|c|c|c|c|c|c|c}
\hline & Nootka & $\begin{array}{c}\text { Amur } \\
\text { Nivkh }\end{array}$ & $\begin{array}{c}\text { Sakhalin } \\
\text { Nivkh }\end{array}$ & $\begin{array}{c}\text { Western } \\
\text { Abenaki }\end{array}$ & Miami & Cree & Wiyot & Yurok \\
\hline North Wakashan & $33 \%$ & $15 \%$ & $16 \%$ & $9 \%$ & $12 \%$ & $11 \%$ & $19 \%$ & $17 \%$ \\
\hline Nootka & & $12 \%$ & $13 \%$ & $10 \%$ & $12 \%$ & $12 \%$ & $9 \%$ & $15 \%$ \\
\hline Amur Nivkh & & & $89 \%$ & $19 \%$ & $18 \%$ & $12 \%$ & $14 \%$ & $23 \%$ \\
\hline Sakhalin Nivkh & & & & $18 \%$ & $17 \%$ & $15 \%$ & $16 \%$ & $22 \%$ \\
\hline Western Abenaki & & & & & $56 \%$ & $61 \%$ & $27 \%$ & $27 \%$ \\
\hline Miami-Peoria & & & & & & $67 \%$ & $35 \%$ & $30 \%$ \\
\hline Cree (Fort Severn) & & & & & & & & \\
\hline Wiyot & & & & & & & $34 \%$ & $25 \%$ \\
\hline
\end{tabular}

${ }^{4}$ Since Proto-North Wakashan is considerably younger than Proto-South Wakashan, composition of a 110-item proto-wordlist for this branch is justified and not very difficult. In the case of Nivkh, since there are only two recorded dialects of this language, their binary comparison will not lead to plausible reconstruction of a separate Proto-Nivkh wordlist. Procedures for reconstructing the Swadesh wordlist for Proto-Algonquian, Proto-Algic, and Proto-Wakashan involve too many difficulties; at present, only data from separate attested languages may be considered satisfactory. 
Table 2. Percentage of lexical cognacy between Algonquian-Wakashan languages (50-item wordlist)

\begin{tabular}{|c|c|c|c|c|c|c|c|c|}
\hline & Nootka & $\begin{array}{l}\text { Amur } \\
\text { Nivkh }\end{array}$ & $\begin{array}{l}\text { Sakhalin } \\
\text { Nivkh }\end{array}$ & $\begin{array}{l}\text { Western } \\
\text { Abenaki }\end{array}$ & Miami & Cree & Wiyot & Yurok \\
\hline North Wakashan & $37 \%$ & $21 \%$ & $19 \%$ & $11 \%$ & $14 \%$ & $9 \%$ & $20 \%$ & $18 \%$ \\
\hline Nootka & & $10 \%$ & $11 \%$ & $10 \%$ & $10 \%$ & $9 \%$ & $9 \%$ & $12 \%$ \\
\hline Amur Nivkh & & & $90 \%$ & $27 \%$ & $23 \%$ & $17 \%$ & $12 \%$ & $20 \%$ \\
\hline Sakhalin Nivkh & & & & $25 \%$ & $21 \%$ & $19 \%$ & $14 \%$ & $19 \%$ \\
\hline Western Abenaki & & & & & $66 \%$ & $67 \%$ & $36 \%$ & $36 \%$ \\
\hline Miami-Peoria & & & & & & $67 \%$ & $46 \%$ & $36 \%$ \\
\hline Cree (Fort Severn) & & & & & & & $47 \%$ & $37 \%$ \\
\hline Wiyot & & & & & & & & $55 \%$ \\
\hline
\end{tabular}

\section{ETYMOLOGIZED 110-ITEM WORDLISTS FOR ALGONQUIAN-WAKASHAN LANGUAGES}

\section{ALL 5}

(1) PAW *k'ว:xkV ( k', g) 'all, every' > PW *k'u:xk- > PWS *č'u:čk- > Noo. č'u:čk 'all, everything' • PA *kahk-el-aw-> FSCr. kahk-iń-aw 'all, every'.

(2) PNA *ček'E ( * *', g, q') 'all, whole' (Nikolaev 2015b, \#83) > PN *chik- > NiA. sik(-m), NiS. sik(-m) 'all' • PAlg *č-ej-ak- ( kh,k')>PA*čya:k- 'all, completely'> MiPe. če: $k-i$ 'all'.

(3) PA *mes- 'all, whole' > WAb. mes- $i$ 'all'.

(4) Wi. Par-aw- 'all'.

(5) Yu. $2 i k-i$ 'subsequent occurence, all' (cf. 2ik-o2t 'always').

\section{ASHES}

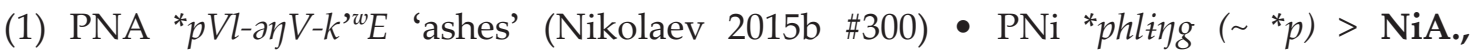
NiS. phling, pling 'ashes' • PAlg *p(el)enekw- ( ph, kh) 'ashes, dust, powder' > PA *penkw- > WAb. sskkwetai-pekwi (lit. “fire dust”); MiP. pinkw-i; FSCr. pihk-ot-e:w 'ashes'. See EARTH, SAND.

(2) PAW *mE: 'flame, fire' (Nikolaev 2015b, \#220) > PAlg *me-hs- 'fire, firewood' > Wi. bac-aw-itk 'ashes'. See FIRE.

(3) $\mathbf{P W N}{ }^{*} G^{w} \partial n$ - 'ashes (of a fire)'> Kw. $G^{w} n-i 2$, Oo. $G^{w} n$-i, Hei. $G^{w} n$-ái, Hai. $G^{w} n-\varepsilon$.

(4) PWS * X'int- > Noo. $\lambda^{\prime}$ int-mis 'ashes' (also Dit. X'idt-ibs 'ashes, dust').

(5) Yu. pont-et 'ashes' (cf. panc-ac 'dust', panc-ah 'be gray (deer)', ponc-ec 'gray deer, white deerskin').

\section{BARK (OF TREE) ${ }^{6}$}

(1) PAW * $\chi e: r g^{w} A \sim{ }^{*} g e: r \chi^{w} A$ 'bark (of tree)' (Nikolaev 2015b, \#397) > PWN * $\chi a: k^{w_{-}}$'bark of tree'> Kw., Oo., Hai. $\chi k^{\prime w}-m$, Hei. $\chi k^{\prime w}-m$.

(2) PWS *c'aq-> Noo. c'aq-mis 'bark of tree' (also Dit. c'aq-abs 'bark of tree; scab').

(3) $\mathrm{PNi}{ }^{*}$ oym $\sim{ }^{*}$ Orm $>$ NiA. oym, o:m, NiS. orm 'bark of tree'.

(4) PAlg ${ }^{*}=l a k w-,{ }^{*}=l e k w-$ 'tree bark' > Yu. $=a r k^{w}-e c, w$-erk ${ }^{w}-e c$; PA *wa=lak-> WAb. wa-lak-a; MiPe. a-lak-i:hk-w-i; FSCr. wa-ńak-e:sk 'bark of tree'.

\footnotetext{
${ }^{5}$ No data for PNW.
}

${ }^{6}$ No data for Wiyot. 
4. BELLY

(1) PAW *2Vta:gA 'belly, abdomen' (cf. Nikolaev 2015b, \#40) > PW *ta:k- > PWN *tok'belly' > Kw. Oo. $t k-' i$, Hei. $t k-’ i$ 'belly', Hai. $t k-' i$ 'belly, abdomen' • PWS *ta(:)̌c- > Noo. ta:čca

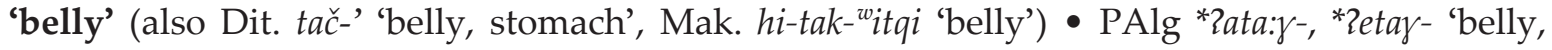
stomach' > Wi. tay-ad-a?l 'one's belly'.

(2) PNi * $\eta(=) \dot{t} m>$ NiA., NiS. $y \dot{z} m$ 'belly'.

(3) PAlg ${ }^{*}=0: d-,{ }^{*}=a d-,=e d-$ 'belly, body' > Yu. =ey-ah 'belly, stomach; PA *=o:t-ay- 'belly' > MiPe. $m-u: t-a y-i$ 'stomach, belly; pouch' and PA *at-ay-, ${ }^{*}-a \check{c}-y-{ }^{*}$ '-eč- $y$ - 'belly; whole body' > FSCr. $m i=s t$-at-ay '(big) belly, stomach'.

(4) PA *-takeš- $y$ - 'guts, intestine' > WAb. =lakes-i 'belly'.

5. BIG

(1) PNA *p'i:lV ( e:) 'big' (Nikolaev 2015b, \#305) > PNi *pil- 'big' > NiA. pil-a-, NiS. pil-d, pil-a 'big; grown up' • PAlg *pel- > Yu. pel-, pl-, popol- 'big'.

(2) PWN *-ka:s 'big, mighty, holy'> Oo. -kas, Hei. -kas, Hai. -kas id., Kw. -kas 'really'.

(3) PWS *2i: $\chi^{w}>$ Noo. 2i: ' 'big' (also Mak. 2i: $\chi^{w}-$, Dit. 2i: $\chi$ ).

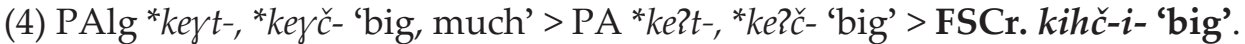

(5) PA *mert- 'big' > WAb. mss-i; MiPe. mehš-i- 'big'.

(6) Wi. dat- 'to be big, large'.

6. BIRD (SMALL, SINGING)

(1) PAW ${ }^{*} c^{\prime} \ddot{a}: q^{\prime w} A,{ }^{*} c^{\prime} V: c q^{\prime w} A$ 'bird (small)' (Nikolaev 2015b, \#77) > PWN *c'asq ${ }^{w_{-}}$( c) 'any small songbird' > Kw. c’osq ${ }^{w}-a ́ n a$, Hei. c'sq ${ }^{w_{-}}, c s q^{w_{-}} \bullet$ PAlg ${ }^{*} c u c k$ - > Wi. cúck-iš, Yu. c'uc'-iš 'bird (small, generic)'

(2) PAW *3i:pV *3i:pV 'bird (small)' (Nikolaev 2015b, \#417) > PNi *cev-rq > NiA. cev-rq, NiS. tev-řq 'bird (small)' - PAlg ${ }^{*} c-e \gamma-e p-(\sim ~ c h, p h)>$ PA *si:p-e:hs-y- 'bird (generic)' > WAb. ssip-ess 'bird (generic)'.

(3) PAW *po:lV $\approx{ }^{*} 20: l p V$ 'large bird' (Nikolaev 2015b, \#290) > PAlg *pel-e: $w w->$ PA *pel-e:hš'bird (small?)' > FSCr. piń-e:š-i:š (with two diminutive suffixes) 'bird (generic)'.

(4) PW * ma:t- 'to fly'> Noo. ma:ma:t-i ; ma:t-iq 'bird (small, generic)'. See FLY.

(5) PA *pi:nč-izl- 'to fly into' > MiPe. pihč-it-a 'small bird (sparrow size)'.

7. BITE $^{7}$

(1) PAW *m'a:whV $\approx{ }^{*} h a m$ 'wV 'to eat, bite' (cf. Nikolaev 2015b, \#234) > PW *m'a:- > Noo. $m^{\prime} a$ 'to bite' (cf. the same root in PWS *ma:- $k^{w}$ - 'to close teeth') - PA *ma:-kw- > FSCr. $m a:-k w-a m-e:-w$ TA, ma:-kw-aht-am TI 'to bite'. See EAT.

(2) PAW * q'an3 $V \sim{ }^{*}$ Gan3 $V(\sim c, s)$ 'to eat, bite' (Nikolaev 2015b, \#315) > PNi *haz- > NiA. jaz- (haz- az-), NiS. jaz-d 'to bite, to dig one's teeth'.

(3) PAlg *-(2)ap-, *-(2)ep- 'by tooth, to bite, eat' > Wi. -ap- 'to bite', see EAT.

(4) PA *sak- 'to hold fast' > WAb. sak-a- 'to bite'.

(5) MiPe. si:hs- 'to bite, pinch'.

(6) Yu. teykel-ew- 'to bite'.

8. BLACK $^{8}$

(1) PWN *c'u:t- 'black' > Kw., Oo. c'ut-a, Hei. c’út-a, Hai. c’ùt-la.

(2) PNi *piw-> NiA. piu-la-, NiS. piw-d, piw-la 'black'.

${ }^{7}$ No data for PWN.

${ }^{8}$ No data for Wiyot. 
(3) PA *-hkat-, *-htk- 'charcoal; black' in PA *ma=(h)tk-anšye:w- 'charcoal; black' > WAb. m-kk-as-aw-i 'black'; *ma=hkat- 'black' > MiPe. ma-hk-at-e:-(w)- 'black', FSCr. ma-hkat-e:$w$-is-iw 'to be black'; PA * (h)kat-k- 'charcoal; black' > FSCr. kahk-it-e:-w-a:-w 'black'.

(4) PAlg *wenliza:yw- 'coals, charcoal' > Yu. lo?ay-, lo?oy- 'embers, coals; black'.

(5) Noo. tupkuk 'black'.

9. BLOOD

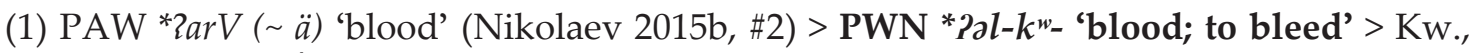
Oo., Hai. ?lo- $k^{w}-a$, Hei. ?lí- $k^{w}-a \bullet \mathrm{PNi}{ }^{*} \eta=a r>$ NiA. $\boldsymbol{y}$-ar 'blood'.

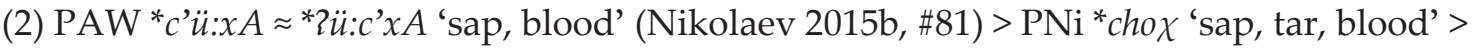

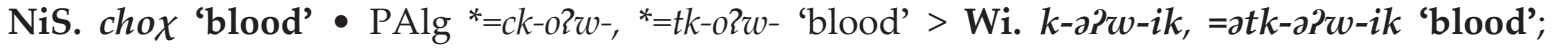
PA * $m e=s k-w$ - > FSCr. $m i=h k-o$ 'blood. See RED.

(3) PAW *p'akV ( ä) 'red; blood' (Nikolaev 2015b, \#302) > PAlg *pak-, *pek- 'to be bloody, red' > Yu. pek-oy(e)k 'blood'; PA *pak-at-kan- > WAb. pak-ak-kan 'blood', PA *ni:-pek- > MiPe. ni:-hpik- 'red; blood'. See RED.

(4) PWS * $x$ is- > Noo. his-mis 'blood'.

10. BONE

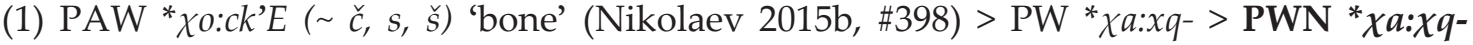

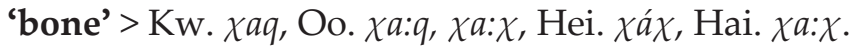

(2) PAW ${ }^{*} t V q^{\prime}\left({ }^{w}\right) E \approx{ }^{*} ? V t q{ }^{\prime}\left({ }^{w}\right) E$ 'bone, gristle' (Nikolaev 2015b, \#213) > PAlg $=\nmid k->$ Wi. =atk-ad-át, Yu. =atk-a? 'bone'; PA *we=tk-an- 'bone; pit' > WAb. o=sskk-an, MiPe. =hk-an-i, FSCr. o-sk-an 'bone'.

(3) PWS *hamu:t > Noo. hamu:t 'bone' (also Dit. habu:t id.).

(4) $\mathrm{PNi}{ }^{*} \eta(=)$ ańfif > NiA. $\boldsymbol{y}$-ińf, NiS. $\boldsymbol{y}$-ańfif 'bone'.

11. BREAST (FEMALE)

(1) PAW *nowV $\approx$ *2onwV 'to suck; breast' (Nikolaev 2015b, \#246) > PWS *2anma 'breast; to suckle' > Noo. Pinma 'breast, milk, sucking milk' (also Mak. 2ada:b(a) 'breast, milk, sucking breast', Dit. 2ama-šs 'breast, milk, breastfeed') - PNi *mo-c (cf. * mo-mo- 'to suck') > NiA. mo-c 'female breast' - PAlg *new- 'breast milk', "new-on- 'to suck (milk)' > Yu. new-on 'breast, nipple, tit, breast milk'; $P A{ }^{*} n o:-n-,{ }^{*} n o:-n-s ̌ y-,{ }^{*}-[n] o:-n$ - 'to suck' > WAb. no-s-ow-o- $k a n$ 'female breast', MiPe. nu:-n-a:-kan- $i$ 'female breast, udder'.

(2) PAlg *-(e)s-en- 'breast, nipple' > Wi. =ás-ad 'breast, nipple'.

(3) PWN * $z a: m$ '- 'breast; to suck at the breast' > Kw., Oo. zam'-a, Hei. zám'-a, Hai. zàm'-a.

(4) NiS. mińk 'female breast' - if not fromńtm- $k$ with metathesis, see (2).

(5) FSCr. či:či:s 'breast; baby bottle'.

12. BURN TR. ${ }^{9}$

(1) PNA ${ }^{*} t u(:)_{b} w V\left(\sim\right.$ o) 'to burn (tr.)' (cf. Nikolaev 2015b, \#345) ${ }^{10}>$ PNi ${ }^{*}$ thuv- > NiA. ruv- (th-), NiS. řuv-nt 'to burn (tr.)' • PAlg *tuw- 'to burn (tr.)' > Wi. tu(w)- 'to burn' (e. g., kita ta tuw-án-izl 'perhaps it is burned out', referring to a canoe). See FIRE, SMOKE.

(2) Yu. tye?w- 'to burn' (e. g., tye?w=ol=ok' 'I burn (trash, brush, etc.)').

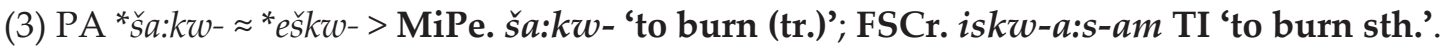

(4) WAb. ccek(a)-, cek(a)- 'to burn (tr.)'.

(5) Noo. $m^{\prime} u$ 'to burn (tr.)'.

${ }^{9}$ No data for PWN.

${ }^{10} \mathrm{Yu}$. tye?w- 'to burn' has been excluded from this comparison. 
13. CLAW, NAIL

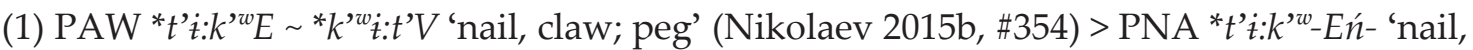
claw' $>{ }^{*} \mathrm{PNi}{ }^{*} t \partial k-(a) n ́ n>\mathrm{NiA}$. tiłk-ń, NiS. tak-(a)ń 'claw, fingernail' • PAlg *-tk-an-(č-ej-),

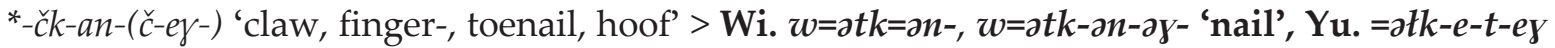
'nail, fingernail, toenail, claw'; PA *=tk-an-š-y-, *=šk-an-š-y- > WAb. =kk-a-s 'claw, nail, hoof', MiPe. $=i-k-a-s ̌-i$ : 'fingernail (of animal or human)', FSCr. $m i=s k-a-\check{s}-i y$ 'finger-, toenail, claw, hoof'.

(2) PWN *c'am-c'am- 'fingernails' (cf. * ${ }^{*}{ }^{\prime} \partial m$ - 'finger(s)) > Kw. c'mc'm-xc'ani?, Oo. c'mc'm-xsk'ana, Hei. c'móc'mí-Gmi.

(3) PW *k'at- > PWS * č'at-ač'a 'finger-, toenail' > Noo. $\check{c}^{\prime} a t-\check{c}^{\prime} a$ 'finger-, toenails' (also Mak. čat-a:č'(a), Dit. č'at-ač').

14. CLOUD

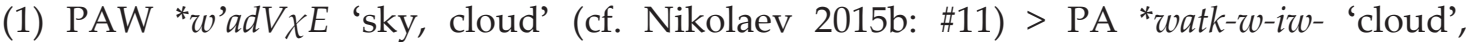

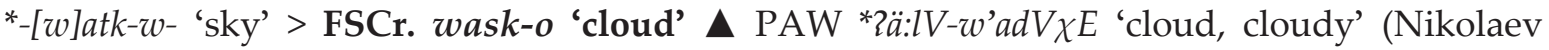

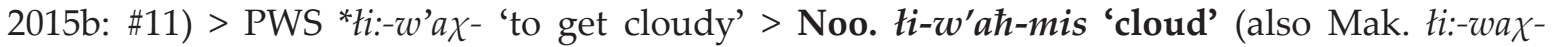
'to get cloudy', Dit. ti:-wax(-k)-, Noo. ti-w'ah- 'cloudy') • PNi *l-ax> NiA., NiS. l-ax 'cloud' • PAlg *a:l-adek-w-, *a:l-edewk-, *al-edwk-w- 'cloud; shadow'> PA *al-etk-w- 'cloud'> WAb.

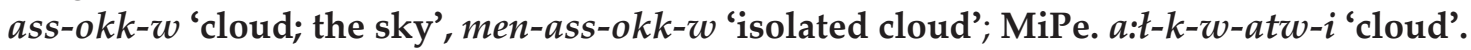

(2) PAW *2VwO:nV ( ך) 'cloud, fog' (Nikolaev 2015b, \#54) > PWN *2an- 'cloud' > Kw. ?n-w-i, Hei. $2 n-u ́ .-i$, Hai. $2 n-u-\grave{\varepsilon}$ 'cloud'.

(3) PAlg. *lop-t-, *lop-č-, *lep-t- (cf. Yu. lohp-izt 'clouds gather, it is cloudy') > Wi. lapt-a?w-, lapč-a?y-, Yu. lept-en-ok'cloud'.

15. COLD ${ }^{11}$

(1) PAW *X'i̇:rqE 'cold' (Nikolaev 2015b, \#208) > PA *tahk-, *tehk- 'cold, cool' > WAb. ttekk-, MiPe. tahk- 'cold', FSCr. tahk-is-iw AI 'to be cold, cool, to cool off'.

(2) PWS * $m$ 'at- 'cold' > Noo. $m^{\prime} a t-u k$ 'cold' (also Mak. bat-, Dit. bat-a:t).

(3) $\mathrm{PNi}{ }^{*}$ civ- $\left({ }^{*} t-\right)>\mathrm{NiA}$. tiv-la, NiS. tiv-d, tiv-la 'cold'.

(4) Yu. sa:w- 'cold' (e. g., sa:w-el-ek' 'I'm cold', sa:w-onc-ek' 'I cool sth. off', etc.)

16. COME

(1) $\mathrm{PW}{ }^{*} \mathrm{Gi}$ :- 'to move; come' > PWN *Gi:- 'to come'> Kw. Gi-la, Oo. Gi-na, Gi-ana, Hei. Gí-na 'come!', Hai. G-an'a-kw 'to come'.

(2) PA *py-a:-/*py-e:- ("py- 'hither' + zero-root 'to go') > WAb. pa-iy-õ AI 'he comes'; MiPe. pya:-; FSCr. pe:-či-:t-oht-e:w AI 'to come'.

(3) PNi * phro- > NiA. phro-, pro-, NiS. phřa-d 'to come, arrive, come near'.

(4) Noo. hin- 'to come'.

(5) Wi. ?uw - 'to come'.

(6) Yu. nes (nesk ${ }^{\left.w_{-}\right)}$'to come, arrive, return'.

17. DIE ${ }^{12}$

(1) PAW *ń'AbV ( p') 'to die' (Nikolaev 2015b, \#255) > PA *nep- 'to die; sleep' > MiPe. nep-e- 'to die, be dead', FSCr. nip-iw 'to die'.

(2) PNA *mo:ryV ( m') (Nikolaev 2015b, \#227) > PNi * $m u-,{ }^{*} m u j->$ NiA. $m u-$, NiS. $m u-d$ 'to die, perish, disappear' (cf. NiA. muj-i-, NiS. muj-vu-d 'sick, to become sick') • PAlg *ma:hy-

11 No data for PWN and Wiyot.

12 No data for PWN. 
'to die', 'to kill' > Yu. moy-k- 'to die'; PAlg caus. *mah[y]-t- > WAb. mac-c-in-a, mac-c-ihl-a 'he dies' (secondary medio-passive).

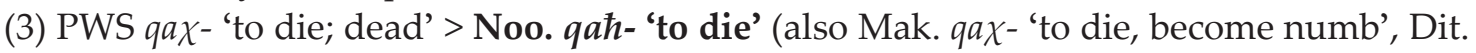
qax-ši $\chi$ - 'to die', Noo. qash-'dead, broken down, beaten, kill').

(5) Wi. $d-a k^{w}$ 'is so, looks so, seems so, happens so, dies' (tabooing).

18. DOG

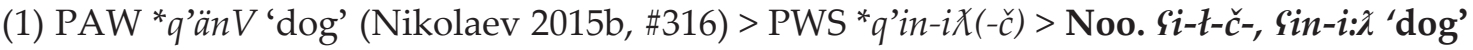

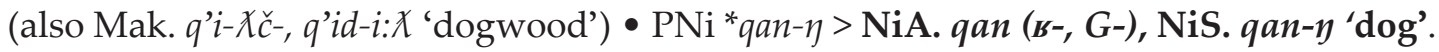

(2) PAW *w'a:yV 'to bark (dog); dog' (Nikolaev 2015b, \#383) > PWN *w'a:-c- 'dog' > Kw. w'a-s-a, He. w'a-c- id., Hai. w'a-c- 'dog or any other quadruped' - PAlg *way-13 > Wi. wáy-ic 'dog'.

(3) PNA *2aLVmV ( $\left.\ddot{a}, m^{\prime}\right)$ 'dog' (Nikolaev 2015b, \#1) > PA *ałem-w- 'dog' > WAb. alem-oss, MiPe. alem-w- $a$, FSCr. atim 'dog'.

(4) Yu. c'iš-ah 'dog'.

19. DRINK

(1) PW *n'a:q- > PWN *na:q- 'to drink' > Kw., Oo., Hai. naq-a, Hei. náq-a 'to drink, to swallow a liquid'; PWS *naq-> Noo. naq-a $\lambda$ 'to drink' (also Dit. daq-šiX id.).

(2) PAlg, PA *men-, -[m]en- 'to drink' > Wi. w-ad-ač-it '3 Sg. drinks sth.' (cf. tar-mad-ač-ir 'water', Yu. men-ok ${ }^{w}$-olum-ek' 'I swallow, gulp down'); PA > MiPe. men- 'to drink sth.', FSCr. min-ihkw-e:- w 'to drink'.

(3) PNi *ta- > NiA. ra- (t-, d-), NiS. ra-(n)d 'to drink'.

(4) WAb. kõkatossmo AI 'he drinks'.

(5) Yu. Pahsp- 'to drink'.

20. DRY (ADJ.)

(1) PNA *Ci(:) ( e[:]) 'to dry' (Nikolaev 2015b, \#82) > PNi *che- 'dry, to dry'> NiA. che 'dry' • PAlg *ce(:)- ( ch, č, čh $)>$ Yu. ce-Pl- 'dry'.

(2) PA *pa:nkw- 'dry' > WAb. pakkw-s- 'dry', FSCr. pa:hkw-a:-w II 'to be dry, be dry land'.

(3) PAlg *ba:2t-, *ba:2c-, *berc- 'dry'> Wi. boc- 'dry' (e. g., bac-ad '3 Sg is dry', etc.); PA *pa:2t-, *pa:2s- > MiPe.pa:hs-i- 'be dry (as by heat or the sun)'.

(4) PWN *lamx $x_{-}$'dry' > Kw. $\operatorname{lm}_{0} x^{w}-a$ 'to be dry, to dry; thirsty', Oo. $\operatorname{lm}_{o} x^{w}-a$ 'dry (enough to be ironed)'.

(5) PW * $x a m s-$ 'dry' > Noo. hapc 'dry, free of wetness'.

(6) $\mathrm{PNi}$ * qhaw- 'dry, to dry' > NiS. qhaw 'dry'.

21. EAR

(1) PAW *hA:t'V $\approx{ }^{*} 2 A h d V$ 'ear' (cf. Nikolaev 2015b, \#142) > PAlg =ehd-(l-), * =ahž- $(r-)$ 'ear' Wi. =atb-o-l-úk, Yu. cp(ey)-a-r 'ear'; PA *hth-aw-ak- 'ear' (cf. suff. *-eht- 'by ear') > $\mathrm{WAb} .=t t-a w-a k-w$, MiPe. $=h t-a w-a k-i$ 'ear', FSCr. $o=h t-a w-a k-a y$ 'her/his ear'.

(2) PAW *no: to hear' (Nikolaev 2015b, \#244) > PNi *no-s > NiA. no-s 'ear'.

(3) PAW *2amE-lV 'ear' (Nikolaev 2015b, \#221) > PNi *m-la > NiS. m-la 'ear'.

(4) PW *p'asp'ay- 'ear' > PWN *p'ssp'ay- 'ear' > Kw. p'spy-’u, Oo. p'sp'i-\{u, Hei. p'sp'i-\{ú, Hai. p’sp'i-u; PWS *p'ap'i-\{i:-> Noo. p'ap'i:- 'ear' (also Mak. p’ip’i-2i:, Dit. p’ip’i-z(i:)).

\footnotetext{
${ }^{13}$ Cf. PA * $[w] a y-,=a y-$ 'dog'.
} 


\section{EARTH}

(1) PAW ${ }^{*} c^{\prime} A k^{w} V \approx{ }^{*} h A \check{c}^{\prime} k^{w} V$ 'earth' (Nikolaev 2015b, \#92) > PW ${ }^{*} c^{\prime} a q^{w_{-}}>$PWN ${ }^{*} c a q^{w_{-}}$, *caq-, * $3 \partial q^{w_{-},}{ }^{*} c^{\prime} \partial q^{w_{-}}$'earth, soil' > Kw. $z q^{w_{-}-a}$ 'earth, soil, etc.', Oo. $c q^{\prime}-m_{o} s$ 'soil', Hei. c'q'-ms 'soil', Hai. cq ${ }^{w}$-al's 'muddy road' - PWS ${ }^{*} c^{\prime} a k^{\prime w_{-}}>$Noo. c c'ak'-umc 'earth, dirt, dust' • PAlg *he:čk-, *hatk-, *hečk- 'earth, land' > Yu. tk-et 'land, ground, clay, dirt' (cf. hełk- 'on land, in the mountains'); PA *atk- 'earth, land' > MiPe. ahk-ihk-iw-i 'earth, soil, ground, land'; FSCr. ask-iy 'land, earth, country'.

(2) PAW *m'e: $\approx$ *2e:m’V 'earth, land' (Nikolaev 2015b, \#238) > PNi *mi-f > NiA., NiS. mi-f 'earth, soil, place'.

(3) PNA * $p V l-\partial \eta V-k^{\prime w} E$ 'ashes' (Nikolaev 2015b, \#300) > PAlg * $p(e l) e n e k w-(\sim p h, k h)$ 'ashes, dust, powder' > PA *penkw- > WAb. pekw-i 'earth, soil, sand, dust'. See ASHES.

\section{EAT}

(1) PAW *m'awhV $\approx{ }^{*} h a m ' w V$ (cf. PA *amw- 'to eat'; cf. Nikolaev 2015b, \#234) > PW *ham'w$>$ PWN *ham'- 'to eat' > Kw. hm-sa, Oo. ham-xzíd, Hei. hing-sa id.; PWS *haw'- > Noo. haw'-a 'to eat' (also Mak. har-ukw id.) • PA *mo:h-> WAb. moh-a TA 'to eat'. See FISH.

(2) PAW *mi:- 'food, fish; to eat' ${ }^{14}>$ PAlg *mi:- > Wi. bi-w- 'to eat; food, fish'; PA *mi:'to eat, food' > WAb. $m i-c-c-i$ AI, $m i-c-i$ TI, MiPe. mi-l-čc-i- 'to eat', FSCr. mi:-č-iw AI+o 'to eat (sth.)'. See FISH, MEAT.

(3) PNA * nit: ( n') 'to eat' (Nikolaev 2015b, \#253)> PA *ni-> NiA. iń- (ńi-), NiS. iń-d 'to eat'.

(4) PAlg *(2)ap-, *-(2)ep- 'by tooth, to bite, eat' > Yu. $n$-ep- 'to eat' (cf. $k^{w}$ oy-p-ey-ok' 'I eat slowly', ternp-ey-ok' [ternp-<*ten-2p-] 'I eat too much', etc.). See BiTe, ToOTH.

\section{EGG}

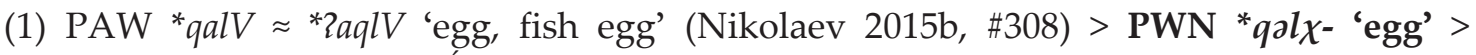

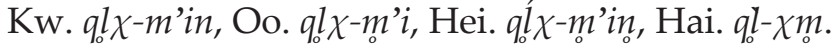

(2) PAW *y'ü:yV 'egg' (Nikolaev 2015b, \#274) > PNi * yoj-eq > NiA. yoj-(e)q, NiS. yoj-q 'egg'.

(3) PNA *2a:wV ( w') 'egg, brood' (Nikolaev 2015b \#21) > PAlg and PA *w-a:w-, *a:w'egg; round' > WAb. $w$-õw-an 'egg'; MiPe. $w-a: w-i$ 'egg (of bird or turtle)'; FSCr. $w$-a:w 'egg'. See ROUND.

(4) Noo. $n^{\prime} u \check{c}^{\prime}-a k$ 'egg' (also Mak. $d u \check{c}$ '-ak ${ }^{w}$, Dit. $d u c ̌-a k$ id.).

(5) Wi. wasad-a?l Pl. 'her/his eggs'.

(6) Yu. Pwayt 'egg (of bird)'.

25. EYE

(1) PAW *2A:sV 'face' (Nikolaev 2015b, \#4) > PWN *G-əs- 'eye’ > Kw. Gy'aGas, Oo. GiGiqs, Hei. qqs, Hai. GGs; PWS *qas-> Noo. qas-i: 'eye' (also Mak. qaš-, Dit. arch. qas-iz).

(2) PAW *n'e: $(w V)$ 'to see, look' (Nikolaev 2015b, \#256) > PNi *na- $\chi>$ NiA., NiS. ńa- $\chi^{15}$ 'eye'. See SEE.

(3) PAlg *=li:n- 'eye' > Wi. =olid, Yu. =elin 'eye'; PA *=šk-[l]i:n-šekw- 'face, eye(s)' > WAb. $=s s-i$-sekw 'eye, face; mask', MiPe. $=h k$-i:n-šikw-i, FSCr. mi-sk-i:-šik 'eye'.

26. FAT (N.)

(1) PNA * $\eta O k^{w} A\left(\sim \eta^{\prime}, q^{w}, X\right)$ 'fat, grease' (Nikolaev 2015b, \#265) > PNi * $\eta 0 \chi>$ NiA., NiS. yox 'fat, lard'.

${ }^{14}$ This new root has been separated from PAW *m'awhV $\approx$ *ham'wV (Nikolaev 2015b, \#234).

${ }^{15}$ Cf. similar suffixal derivative in PWS $n$ ' $a-\check{c}^{-}$'to look' $<\mathrm{PW}{ }^{*} n$ ' $a-k$-. 
(2) PAlg *w(-ej)-el- 'fat' > Wi. du=wal-ákh"s-apl-aw 'my (animal's) fat', Yu. wel 'fat'; PA *wi:l-en-w-> MiPe. wi:l-in-w-i 'fat'.

(3) PAlg *pem-ey- 'grease, oil' 16 > PA *pem-y- > WAb. -pem-i 'fat'; FSCr. pim-iy 'grease, fat, oil'.

(4) PWN * $\chi^{\prime} a$ :-s 'fat, grease, oil, blubber' > Oo. X'a-2s 'animal fat, etc.', Hei. X'á-s(-) 'oil, grease, fat', Hai. X'a:-s 'animal fat, etc.'.

(5) Noo. גaq-mis 'fat, grease, oil'.

27. FEATHER

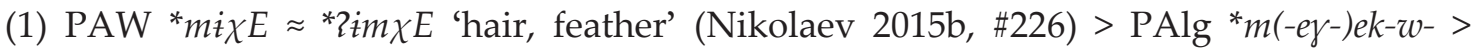
PA *mi:k-w-an-> WAb. mik-w-en 'feather, quill, pen', FSCr. mi:k-w-an 'feather'.

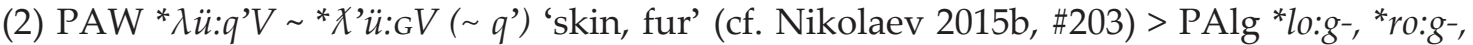
*reg- 'skin, feather' > Yu. re?-n-oh 'feather'.

(3) PWN *c'alk- 'feather(s)' > Kw. c'lc'lk 'feathers', Oo. c'ac'lk-'a 'to get feathers', Hei. c'lg-m 'long feather', Hai. c'lc'lk 'feathers'.

(4) PNi *tup-r > NiA. tup-r, NiS. tup-ř 'feather(s), down'.

(5) Noo. Giya:t 'feather'.

(6) MiPe. ahsawa:nkatia '(large) feather'.

(7) Wi. wal-, -awal- 'feather' (in wat-wal-at '(swallowing) feather', 2al-awal-ap-ti? 'what kind of feathers').

28. FIRE ${ }^{17}$

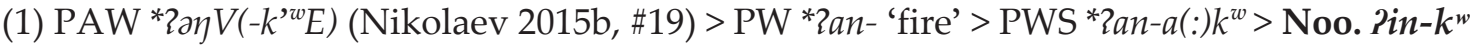
'fire, burning' (also Mak. 2ad-a:k ${ }^{w}$, Dit. ?ad-ak 'fire').

(2) PAW * $m E$ : 'to flame, fire' (Nikolaev 2015b, \#220) > PAlg *me-hs- 'fire, firewood' $18>$ Wi. bo-s; Yu. me-c 'fire'. See ASHES.

(3) PNA *tu(:) $)_{w} w(\sim o)$ 'to burn (tr.)' (cf. Nikolaev 2015b, \#345) > PNi *thuf-r > NiA. thuy-r, thu:-r, NiS. thu -r $^{\prime}$ 'fire'. See BURN, SMOKE.

(3) PA *eškw-et- 'fire' (cf. PA *ša:kw- $\approx$ *eškw- 'to burn') > WAb. sskkw-et-a, MiPe. kot-e:w-i, FSCr. išk-ot-e:w 'fire'. See BURN.

29. FISH

(1) PAW * ว̌u: 'fish, salmon' (Nikolaev 2015b, \#420) > PNi *cho > NiA. cho (s-), NiS. cho 'fish'.

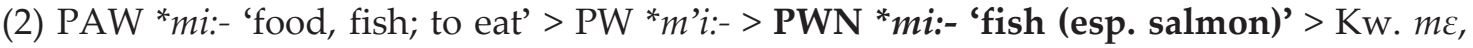
Hei. mi-z, Hei. mi-á, Hai. màmi-a • PAlg *mi:- 'to eat, food'> Wi. $\boldsymbol{b i}$ - $\boldsymbol{w}$-i? 'food, fish'. See EAT.

(3) PAW *m'a:whV $\approx$ *ham'wV 'to eat' (Nikolaev 2015b, \#234) > PW *ham'w- 'to eat' > PWS *haw'- > Noo. ha?-um 'fish, food' (cf. har-uk'to eat'). See EAT.

(4) PNA *lOńmV ( l', n', m') 'salmon, trout' (Nikolaev 2015b, \#194) > PAlg *nam-, *nem'trout, sturgeon; fish' > Yu. nep-e?w-iš 'fish (generic)' 19; PAlg *nam- > WAb. nam-as 'fish'.

(5) PNA * ga: $V V$ 'salmon, trout' (Nikolaev 2015b, \#109) > PA *ken-, *kiko:n-> MiPe. ki:hkon$e: h s-a$; FSCr. kin-oš-e:-w 'fish (generic)' .

${ }^{16}$ Cf. Yu. pem-ek' 'I cook'.

17 No data for PWN.

${ }^{18}$ Cf. PA *me-ht- 'firewood'.

${ }^{19} \mathrm{Yu}$. -p- by analogy with *nep- 'water', * $n$-ep- 'to eat'. 
30. FLY (VB.)

(1) PAW *n'o:lV 'to fly' (Nikolaev 2015b, \#249) > PAlg *-[n]a:l- > Yu. -ol- 'to fly' (e. g., s-ol-ek', wen-ozom-ol-ek' 'I fly', etc.); Wi. -al-, -ar- 'to fly' (e. g., tal-al-ál-it 'she is flying around (there)', kaw-ar-ar-áš-it 'they start flying around in a flock', etc.); PA *pem-izl- 'to fly along' > WAb. pem-it-õ- $k$ II 'it flies'; FSCr. pim-iń-a:- 'to fly'.

(2) PW *ma:t- > PWN *ma:t- 'to fly'> Kw. mat-, Oo. mat-la, Hei mát-lá; PWS *mat- > Noo. mat- 'to fly'.

(3) PNi * $p i j->$ NiA. $p i j-$, NiS. $p u j-d$ 'to fly'.

(4) MiPe. ampahwi- 'to fly'.

31. FOOT

(1) PAW * $g^{w} i: g^{w} V$ foot' (Nikolaev 2015b, \#117) > PW * $g^{w} i: g^{w} i$ : 'foot, leg, flipper' > PWN ${ }^{*}$ gu:gwi:- 'foot, leg' > Kw. gug $g^{w} y$-'u, Oo. $g^{w} u g^{w} i$, Hei. $g^{w} u^{w} g^{w}$, Hai. gùg ${ }^{w} i$.

(2) PAW *či:t'V(-lV) 'foot, leg, flipper' (Nikolaev 2015b, \#86) > PAlg *-cit(-t-) 'foot'20 > Wi. huw=elit-apl 'her/his foot'; PA *=sit- 'foot' > WAb. =sit 'foot (body part)'; FSCr. mi-sit 'foot, paw'. See TAIL.

(3) PNA *2VčkE ( q,X) 'foot, leg' (Nikolaev 2015b, \#39) > PNi * $\eta=a c x>$ NiA. $y$-ic $x$, NiS. y-acx 'foot, leg' • PAlg *-tk-a:t-, *-čk-a:č- 'foot, leg' > Yu. =ack-ah 'foot'; PA * =tk-a:t-21 > MiPe. $=h k-a: t-i$ 'foot'

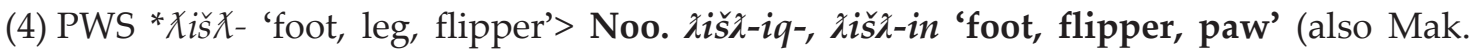
Xizišč-aq-, Xizišč-id(-a) 'foot, feet, leg, fish tail, whale fluke').

32. FULL

(1) PAW *クü:šV 'full' (Nikolaev 2015b, \#267) > PA *mo:š-k-en-, *-[m]aš-k-en- 'full' > MiPe. mo:h-k-in-eč-i:- AI 'to be full', FSCr. sa:k-ašk-in-e:p-e:w 'to be full (of liquid)'.

(2) PAW ${ }^{*} q^{w} 0: K t V$ 'full' > PWN * $q^{w} u: t^{\prime}-$ 'full' > Kw. qut'-a, Oo. $q^{w} u t$ '-a, Hei. $q^{w} u t^{\prime}-a$, Hai. qùt'-a $\bullet$ PAlg [*koht-], *kohc- > Yu. kohc- 'full' (in kohc=eww=et 'to be full', etc.)

(3) PNi *char- 'full, to fill' > NiA. char- 'full'.

(4) PNi *ta-ta- 'whole, entire, to care' > NiS. ta-ta-d 'full'.

(5) Noo. cuma: 'full'.

(6) WAb. pessan- 'full'.

(7) Wi. -esw- 'to be full'.

33. GIVE

(1) $\mathrm{PAW}{ }^{*} c^{\prime} \mathrm{O}: \gamma^{w} V \approx{ }^{*}\left\{O: \gamma^{w} \xi V\left(\sim b^{w}\right)\right.$ 'to give' (cf. Nikolaev 2015b, \#95) > PW * ${ }^{*}$ 'u:- 'to give' PWN *c'u:- 'to give' > Kw. c's, Oo. c'u-a, Hei. c'u-á • PA*?o(:)z-> Yu. ?o? 'to give'.

(2) PNA *me: $\approx{ }^{*} 2 e: m V\left(\sim m\right.$ ') 'to give' (cf. Nikolaev 2015b, \#225) > PNi *kh-im-, ${ }^{*} i m-k h->$

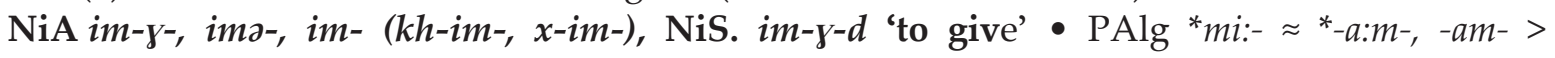
Wi. - $\partial \boldsymbol{b}-,-\boldsymbol{a b}$ - 'to give' (e. g. $2-\partial c-\partial b$ - 'to give it to', yac-ab-um 'I give to smbd.', etc.); PA *mi:-l(cf. Wi. bi-l- 'to divide and distribute') > WAb. mi-l- 'to give (sth.)'; MiPe. mi:-l- 'to give (to him)'; FSCr. mi:-ń-e:-w TA 'to give (sth. to so.)'.

(3) PWS *-ay-i: 'to give' > Noo. hin-i: 'to give' (also Mak., Dit. hid-i:).

34. GOOD

(1) PW *2i:k- 'good, fine' > PWN * $i$ :k- 'good, nice, well, fine, causing satisfaction' > KW., Oo., Hei, Hai. ?ik.

${ }^{20} \mathrm{PA}^{*}$-sit- and secondary *-XiX-in Wi. -elit 'foot'.

${ }^{21}$ Cf. suff. *-ešk-, *-ehk- 'by foot or body'. 
(2) PW *Xu:t- 'good, even'> Noo. zut 'good, pretty' (also Mak. Xut-(u:) 'clean, good', Dit. Xut' 'good, pretty').

(3) PNi *nama- > NiA nama-, NiS. nama-d 'good, perfect, industrious'.

(4) PAlg *ko:c-, *keč-22 > Wi. kuc 'good, well'.

(5) PA *wal-, *wel-23>WAb. wal-i, ol-i- 'good'.

(6) PA * melw- 'good, fine, beautiful' > FSCr. minw- 'good'.

(7) MiPe.nah(i)- 'good'.

(8) Yu. skew-, sku- 'good, well'.

35. GREEN ${ }^{24}$

(1) PAW *quä:lV *Gwä:lV( Ł) ‘blue, green’ (Nikolaev 2015b, \#325) > PNi *qala- > NiA qala'green, unripe'.

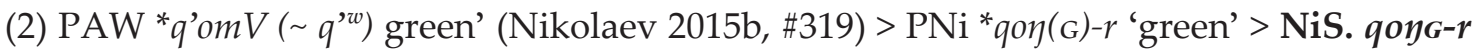
vala-d 'green' (with $\mathrm{PNi}$ *val 'colour').

(3) PAlg *wark-, *werk- 'green, verdure' $25>$ PA *wašk-, *wešk- 'green, raw' > WAb. asskkasskk-w-i 'green'; MiPe. ihk-ip-an- II 'to be blue/green'; FSCr. oša:-wašk-os-iw AI, oša:$w a s ̌ k-w-a: w$ II 'to be blue-green'.

(4) PW ${ }^{*} t i: \chi^{w}{ }_{-},{ }^{*} t u: \chi^{w}{ }^{w}$ 'evergreen; bile' > PWN ${ }^{*} t i: \chi$ - 'green' > Kw. ti $\chi-a$ 'hemlock leaves, leaves of evergreen', Oo. ti $\chi$-sm 'green rock', Hai. tì $\chi$-sdu 'green, yellow'.

(5) Noo. kist-aq 'green, pale'.

36. HAIR (HEAD-)

(1) PAW *həpV(-lV) 'hair (body, facial)' (Nikolaev 2015b, \#145) > PW *hap- 'hair (body, facial)' > Noo. hap, hap-sy'up 'hair' (also Mak. hap- 'hair, fur', Dit. hap-a:b-\{ub 'hair') •

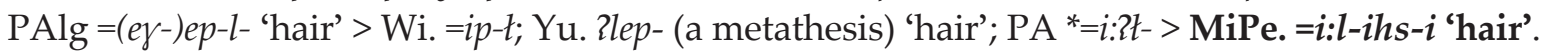

(2) PAW *mi $\chi E \approx *$ *im $\chi E$ 'hair, feather' (Nikolaev 2015b, \#226) $>$ PNi * $\eta=a m x>$ NiA $\boldsymbol{y}$-ing, NiS. $y$-amx 'head hair, animal hair'. See FEATHER.

(3) PNA *2O:čk'E 'head, face' (Nikolaev 2015b, \#36) > PAlg * ${ }^{*} a: t k w-,{ }^{*}=e t k w-$ 'head, hair' > $\mathrm{WAb}$. =tep- $k k w$-an 'head hair'.

(4) PW *-qi: 'head' > PWN *-qi: 'head, top of head, hair of head'> Oo. $-(:)-q i(-a)$, Hei. -(:)-qi-(a), Hai. -(:)-qi-(a).

(5) PA * =pi:w- 'plume, down, short feather' > FSCr. mi-pi:w-ay 'hair'.

37. HAND

(1) PAW ${ }^{*} d i: m V_{G}{ }^{w} E\left(\sim q^{w w}\right)$ 'arm, hand' (cf. Nikolaev 2015b, \#103) > PNi *tamk > NiA timk, NiS. tamk 'hand, arm'.

(2) PAW ${ }^{*} n^{\prime} O L K\left({ }^{w}\right) V(\sim$ n') 'arm, hand' (Nikolaev 2015b, \#250) > PA *=netk- 'arm' > MiPe. $=n e h k-i$ 'hand; fingers'.

(3) PAlg * $=(e)$ 2s- 'hand' > Wi. =é?s; Yu. cew-es 'hand'; PA =2t-en-ty- > WAb. =el-t, =el-c-i 'hand'; FSCr. $m i-\check{c}-i h-\check{c}-i y$ 'hand, foreleg of animal'.

(4) PWN *ha:y'a:-su: 'hand' > Kw. hay'asú, Hei. háy'ásu, Hai. hay'asu, harisu.

(5) Noo. $k^{w i k} k^{w i n k}$-su 'hand'.

${ }^{22}$ Also PA *keš- $y$ - 'good'.

${ }^{23}$ PA *wel- > Mic. wel-apit-ay 'I have good theeth', etc.

${ }^{24}$ No data for Wiyot and Yurok.

${ }^{25}$ Also Yu. 2wesk-(k)a:p' 'crab grass, green', 2wesk-em 'crab grass, cut and dried'. 
38. HEAD

(1) PAW *hü:xE 'head, nape' (Nikolaev 2015b, \#155) > PWN *hi:x- 'head' > Oo. hix-t'i, Hei. híx-t'i, Hai. hìx-t'i id., Kw. hix.-t'i 'fishhead'.

(2) PAW ${ }^{*} t^{\prime} \dot{t} q^{w} E \sim{ }^{*} t i q^{w} E$ 'head' (Nikolaev 2015b, \#353) > PW *t'u $\chi^{w}{ }^{w},{ }^{*} t^{\prime} u q-$ 'head, forehead'

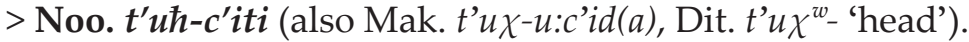

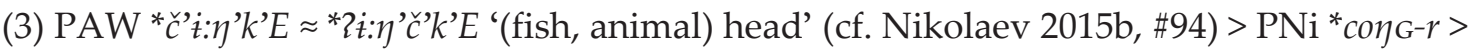
NiA coyr (z-, 3-); NiS. coy-r, coy $-\check{r}$, coyq-r' head' 26.

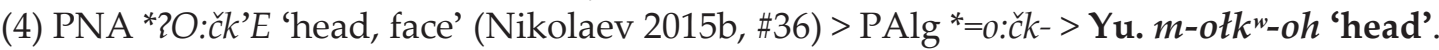

(5) PAlg *temp- 'head, brain' > Wi. w-otb-a(?)t 'head'; PA *temp- 'head, brain' > $\mathrm{WAb} .=$ tep 'head; bowl of a pipe'; MiPe. =ntep-ik-an-i 'head'.

(6) PAlg *we=2t-ekw-a:n-, *-št-ekw-a:n- '(fish) head' (cf. Yu. to:-k-un 'fish, salmon head') > FSCr. $m i$-st-ikw-a:n 'head'.

39. $\operatorname{HEAR}^{27}$

(1) PAW *marV $\approx$ *2am?V 'to hear' (Nikolaev 2015b, \#221) > PNi *mi- > NiA mi-, NiS. mi-d 'to hear, listen' - PAlg * $-o(:) 2 m-,{ }^{*}-o 2 m-,-e 2 m$ - 'to hear, understand' > Wi. $k$-an-i?m-it 'to hear'; Yu. $k-o(?) m$ - 'to hear; understand, feel'; PA *ne-o:m-t-, *pe-em-t- > WAb. $n-o-t-a$ - 'to hear'; MiPe. $n$-o:n-t-am- TI 'to hear, understand'; FSCr. $p$-e:h-t- 'to hear'. See EAR.

(2) PAW *no: 'to hear' (Nikolaev 2015b, \#244) > PW *na:- > Noo. $n a-? a($ :) 'to hear; feel, perceive' (also Dit. da-2a: 'to hear, understand', Mak. da-2a: 'to hear, perceive, sense'). See EAR.

40. HEART ${ }^{28}$

(1) PAW * $2 e p V$ 'heart' (Nikolaev 2015b, \#13) > PNi * $\eta=i f>$ NiA., NiS. $y$-if 'heart'.

(2) PAlg ${ }^{*}=t e k^{w} l-,{ }^{*} c e k^{w} r$ - 'heart' > Wi. w-otw-ihl 'her/his heart'; Yu. cek ${ }^{w} S$ 'heart' (cf. tek ${ }^{w} S-a ? r$ 'heart of salmon, uvula'); PA *te:h-> MiPe. =te:h-i 'heart'; FSCr. $o=t e: h$-iy 'her/his heart'.

(3) Noo. tim'aqsti 'heart; mind; brain; spinal cord; pithy core of any tree'.

(4) WAb. =lawõkan 'heart'.

41. HORN

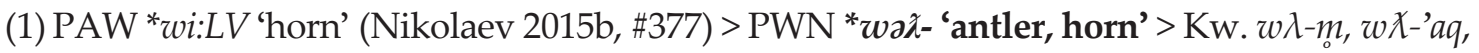
Oo. $w \lambda$-m, Hei. $w \lambda$-mó, Hai. $w \lambda$-'aq • PA *wi:t-, *wiwi:t- 'horn'> MiPe. =wi:wi:la 'horn, antler'.

(2) PAlg * $=a: n-,{ }^{*}=e n$ - 'head hair, horn(s)' ${ }^{29}>$ Wi. =ad-ad 'horn'.

(3) PNi * murk-i > NiA murk-i, NiS. muřk-i 'horn'.

(4) PA *e:šk-an- 'horn' > WAb. asskk-an 'horn, antler'; FSCr. e:šk-an 'antler'.

(5) Noo. ma:t 'horns'.

(6) Yu. s?ec-oh 'horn(s); wedge'.

42. I

(1) PAW *nV 'I; we (excl.)' (Nikolaev 2015b, \#254) > PW *nu:- 'I, we' > PWN *nu:-gwa: 'I' > Kw., Oo. $n u-g^{w} a$, Hei. $n u_{-} g^{w} a$, Hai. $n \grave{u}-g^{w} a \bullet$ PNi *ńi > NiA., NiS. ńi 'I' • PAlg *ne?- (pref.) 'I, me, my', *ne-2il-a 'I' > Wi. yi(-l) 'I' (cf. du- '1 Sg possessive prefix)'; Yu. ne-k(i?); PA *n-i:l-a > WAb. $n$ - $i-a$ 'I, me, mine', MiPe. $n$-i:l- $a$ 'I, me', FSCr. $n$ - $i: n-a$ 'I'. See WE.

(2) PWS *si-y'a: 'I' > Noo. si-y'a 'I' (also Mak. si-ya:, Dit. si-y'a).

${ }^{26}$ In Nikolaev 2015b, \#94 PAlg *-a:čk-w-, *-ečk-, *-etk- ( čh, kh) 'head' [PA *-etkw-, -ešky- (suff.) 'head', Yu. $m$-otk ${ }^{w}$ - 'head'] were erroneously grouped together with PAW *č' $i: \eta^{\prime} k$ ' $E \approx{ }^{*} 2 \dot{i}: \eta^{\prime}{ }^{\prime}{ }^{\prime} k$ ' $E$ 'head'; I now think that they should instead be grouped with PNA *2O:čk’E 'head, face' (Nikolaev 2015b, \#36).

${ }^{27}$ No data for PWN.

${ }^{28}$ No data for PWN.

${ }^{29}$ Also PA * $=a: n$ - 'head hair'. 
43. KILL

(1) PAW * $\chi V l V \approx * 2 V l \chi V$ 'to kill' (Nikolaev 2015b, \#400a) > PWN *2al $\chi$ - 'to kill, murder,

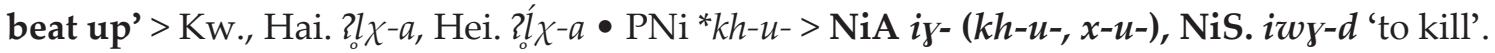

(2) PAW *n' $A b V(\sim p$ ') 'to die' (Nikolaev 2015b, \#255) > PAlg *nep- 'to die', caus. *nep-l'to kill' > PA *ne?-l- > WAb. nih-l-a TA 'to kill (so.)' $\boldsymbol{\Delta}$ *nep- 'to die' > caus. FSCr. nip-ah-e:w TA ‘to kill so.'. See Die.

(3) PWS *qa - 'to die; dead' > Noo. qah- 'to die; kill, cause to die' (also Mak. qa - 'to die,

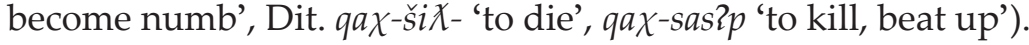

(4) MiPe. ankih- 'to kill (him)'.

(5) Wi. diy-ab- 'to kill'.

(6) Yu. caus. sam-at- 'to beat, kill'.

44. KNEE

(1) PAW * $\chi V t V$ (Nikolaev 2015b, \#401a) > PWN *- $\chi t$-a:m'u: 'knee'> Kw., Hei., Hai. -(:) $\chi t$-am'u - $\mathrm{PA}{ }^{*}=k e t-e k w$ - 'knee' > WAb. $=k-k e t=e k w$; MiPe. $=h-k i t-i k w-i-a$ 'knee'; FSCr. $o=k i t-i k$ 'her/his knee, knee joint'.

(2) PAW *p'iqE 'knee' (Nikolaev 2015b, \#304) > PWS *-p'iq-a > Noo. Pa?ap-p'iq-a 'knee' (also Dit. -p’iq id.) • "pix-> NiA. pix (v-, b-), NiS. pix-t-i 'knee'.

(3) Wi. =a?l-aw-al 'knee'.

(4) Yu. 2o:k-at 'knee'.

45. KNOW ${ }^{30}$

(1) PAW * $k^{w} e: m V$ 'to know, understand' (Nikolaev 2015b, \#177) > PW * $\chi a m^{-31}>\mathrm{PWS}$ * $x a m$-up- 'to know' > Noo. ham-up 'to know, recognize' (also Noo Ky. ham-i:p id., Noo. caus. him.- 'to show', Mak. xab-up 'to know, recognize (a person)', Dit. xab-up 'to know, recognize') - PNi *him-, *khim- > NiA jajm- (him-), jim- (khim-, xim-), NiS. jajm-(n)d 'to know, understand' • PAlg ${ }^{*} k o(:) m-(\sim k h)>$ Yu. kom- 'to know, feel' (e. g., kom-c-um-ek' 'I know', etc.).

(2) $\mathrm{PAW}{ }^{*} h \mathrm{O} \chi V-t A: k^{w} V$ 'to know how' (cf. Nikolaev 2015b, \#35) > PAlg *ka:tkw-, ${ }^{*} k e t k(w)->$ Wi. kak $^{w_{-}}$'to know'; PA *ketk- 'know, recognize' > MiPe. kihk-eli:-, FSCr. kisk-e:ńim-e:w TA 'to know'.

(3) PA *wa:w-, *waw- 'to know' > WAb. waw- 'to know, learn' (e. g., waw-alt-a 'to know (in general), be knowing', waw- $k-a$ 'to know how to dance', etc.).

46. LEAF

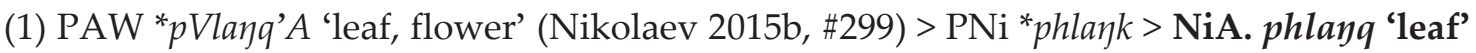
- PAlg ${ }^{*} P(e l) a K-w$ - 'leaf' > PA*-pak-w- > WAb. wani-pakw, MiPe. mihši-pakwa (Mi.), ka:ki-pakwa (Pe., Wea.) 'leaf'.

(2) PAW *mi: ( ä:, ü:) 'leaf, berry' (Nikolaev 2015b, \#224) > PWN *mi:- 'leaf, blossom' > Kw. mame-m'a 'leaves, blossoms', Hei. mím-íax Xawa 'leaves (of any plant)'. 'leaf'.

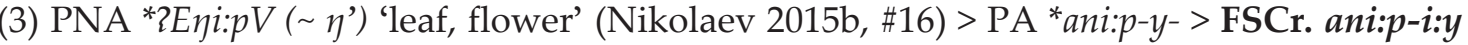

(4) PWS * X'aq- 'to grow; to sprout' > Noo. $\lambda^{\prime} a q$-apt 'leaf; bush; branch; plant'.

(5) $\mathrm{PNi}{ }^{*} \mathrm{com}-r>\mathrm{NiS}$. com-ř 'leaf, flower'.

(6) Wi. Pawiyadak 'plant, grass, weed, leaf'.

(7) Yu. ka:p'- 'leaf, leaves, greenery, brush, grass, the wild; to gather greenery'.

${ }^{30}$ No data for PWN.

${ }^{31}$ Cf. Quil. $\chi a b$ - 'to know how'. 
47. LIE (ANIMATE OBJECTS) ${ }^{32}$

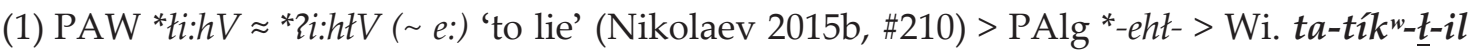

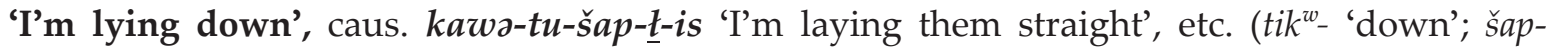
'in the same way'); PA *-eht-, *-ehš- 'to lie, fall' > WAb. pap-is-ew-õč-em-o AI 'he lies'; FSCr. pim-iš-in AI 'to lie, recline'.

(2) PWN ${ }^{*} k^{w}$ al- 'to lie somewhere, lie down (said of animate beings)' $>\mathrm{Kw} . k^{w w} l-a$, Oo. $k^{w} l-a$, Hei. $k^{w} l-a ́$ id., Hai. $k^{w} l-z u \varepsilon \grave{t}$ 'mattress'.

(3) PNi * poř-> NiA. por- (b-), NiS. poz-d 'to lie, lie down'.

(4) Yu. Poytkes-ek' 'I lie (down)'.

48. LIVER

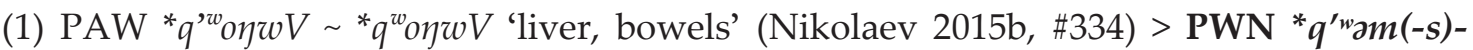

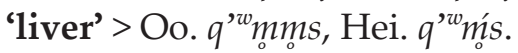

(2) PAW ${ }^{*}$ tij $\left({ }^{w}\right) V$ 'liver, gall' (cf. Nikolaev 2015b, \#341) > PNi *thi-w-s > NiA. thiu-s, NiS. thiw-s 'liver'.

(3) PAW * ${ }^{*} a: q^{\prime w} A \approx{ }^{*}\left\{a: r^{\prime} q^{\prime w} A\right.$ ( a:) 'liver' (Nikolaev 2015b, \#338) > PAlg *=tkw-en-, *=tkw-an$>$ Wi. =at $w$-əd, Yu. =atk-un 'liver'; PA WAb. =skw-en, MiPe. =hk-on-i, FSCr. o-sk-on 'liver'.

(4) Noo. $x^{\prime} i m^{\prime} a(:) q$ 'liver'.

49. LONG

(1) PAW *gil'V ( a) (cf. Nikolaev 2015b, \#113) > PWN *gal'-t- 'long'33 > Kw. gl-t-'a, Oo. gl-t, Hai., Hei. gĺ-t • PNi *kìl- > NiA. kìl-a-, NiS. kìl-d 'long'.

(2) PAlg *ken-ew- 'long' > Yu. kn-ew- 'long, tall'; PA *ken-w-, “kaka:n-w- 'long' > WAb. kwen-i id., MiPe. kin-w-a:- II, FSCr. kin-os-iw AI, kin-w-a:w II 'to be long'.

(3) Noo. $y^{\prime} a(:) q$ 'long, high'.

(3) Wi. dan-at-a?w' '3Sg is long' (cf. dan-at-ad '3Sg is large').

50. LOUSE (HEAD-) ${ }^{34}$

(1) PAW *hi:rxk'E 'louse' (Nikolaev 2015b, \#149) > PW ${ }^{*} G=i: x k$ - 'louse' > PWN *G-i:x'louse' > Kw., Hai. G-i-n, Oo. G-i:-no-, Hei. G-i-na (cf. *G-ix- 'to have lice, lousy'); PWS *q-ič- > Noo. q-ič-in 'louse' (also Mak. q-ič-, q-ič-id(a), Dit. q-ič-id) • PAlg *ihkw- 'louse' > Wi. ík 'louse', Yu. m-ohk-oh 'head louse'; PA *ehkw- > WAb. akk-em-õ; MiPe. at-ehk-am-a 'louse', wa:p-ihkw-a 'louse, lice'; FSCr. ihkw-a 'louse'.

(2) $\mathrm{PNi}$ *amrak > NiA. amrak 'head louse'.

51. MAN ${ }^{35}$

(1) PAW *2ü:tOq'w E (Nikolaev 2015b, \#38) > PNi *utk-> NiA. utk-u 'man, husband'.

(2) PAW ${ }^{*} b e: k^{w} E \sim{ }^{*} p e: g^{w} E$ 'person' (Nikolaev 2015b, \#62) > PAlg *na:-pe: $w^{*}$-, ${ }^{*} n e-p e: \gamma w->$ Yu. pey-ək 'man, mal, all-grown-up person' (cf. pej-it 'male'); PA *na:-pe:w- 'man, male' > FSCr. na:-pe:w 'man'. See PERSON.

(3) PW *kap- 'man, husband' > PWS *čap- $k^{w}{ }^{\text {-, }}$ *čakup- (metath.) > Noo. čakup 'male, husband, man' (also Mak. čap- $x^{w}-$, čakup, Dit. čap- $\chi^{w}-$, čak $k^{w} u p$ 'male, husband').

${ }^{32}$ No data for MiPe. or Noo.

${ }^{33} \mathrm{Cf}$. PWN *gal- in Kw. gl-i2stnd 'to turn over lengthwise (said of salmon, small animal)'.

${ }^{34}$ No data for NiS.

${ }^{35} \mathrm{NiS}$. azmac 'man' is borrowed from TM *āsimač- 'to have an affair with somebody else's wife', ‘āsimaču 'womanizer'. 
(4) PA *elenyiw- > MiPe. aleni-a 'man'. See PERSON.

(5) PWN *wi:s- 'man, male' > Kw. wis-m 'male', wís-a 'small boy', Hei. wís-m 'male', Hai. wìs-m 'man, male'.

(6) WAb. sanõpa 'adult male, man, husband'.

(7) Wi. ku?w-il 'man, Indian, person, human, creature, people' (cf. ku\{w- 'to live'). See PERSON.

52. MANY

(1) PWN * q'i:- 'much, many' > Kw., Oo. q'i-nm, Hei. q'í-nm̆, Hai. q'ì-nm.

(2) PWS *2ay- > Noo. ?ay-a 'many' (also Mak. ?ay-, ?ay-a- 'many, much', Dit. ?ay-, ?ay-i:q 'a lot').

(3) $\mathrm{PNi}$ *les > NiA., NiS. les 'many; enough'.

(4) PA * me?t- 'big' > WAb. mss-al-i 'many, much'.

(5) Wi. kac- 'many, much, a lot'.

(6) Yu. ten- 'many, much, a lot'.

(7) MiPe. wi:hsa 'much, a lot, great amount'.

(8) FSCr. mihče:t, misče:t 'many, a lot, a good number'.

53. MEAT

(1) PAW *di: ${ }^{w} V \sim{ }^{*} d i: G^{w} V(\sim \ddot{a}:)$ 'meat, flesh' (Nikolaev 2015b, \#101)> PNi ${ }^{*} t j u-\check{r}>$ NiA. cu-s (3-), NiS. $t u-\check{r}$ 'meat'.

(2) PAW *mi:- 'food, fish; to eat' > PW *mi:- 'fish, fish meat' > Noo. bi:-c 'meat' (also Mak. bi:-c-i: 'meat', Dit. bi:-c 'meat, flesh'). See EAT, FISH.

(3) PNA *yizv ( y', a, w') 'body, intestines; flesh' (Nikolaev 2015b, \#404) > PA *=i:-yaw'meat; body' > WAb. w-i-o-ss, MiPe. w-i:-yo:-hs-i 'meat', FSCr. $w$-i-ya:-s 'meat, flesh'.

(4) PWN *2alz- 'meat, flesh'> Kw. ?lz-i, Hai. ?lz.

(5) Yu. nopaw 'meat'.

(6) Wi. ̌̌ečač 'meat'.

54. MOON

(1) PAW *l'u:y'zV (Nikolaev 2015b, \#197) > PWN *n'u:?s-i: 'moon, month' > Oo. n'urs-i,

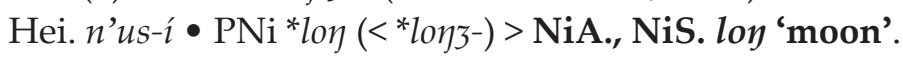

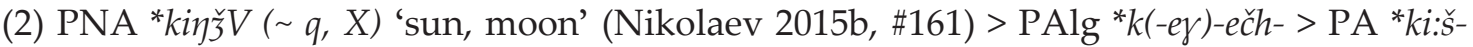
'sun, moon, month; day, sky' > WAb. kis-oss 'moon, sun, luminary'; MiPe. ki:ts-w-a 'sun, moon; month'. See SUN.

(3) Noo. hupat 'sun, moon, month'. See SUN.

(4) Wi. dacaw-alayalakwi 'moon' (dacaw- 'night'). See NiGHT.

(5) Yu. won=ewsley 'moon, sun' (won- 'up'). See SuN.

(6) FSCr. tipisk(a:w)i-pi:sim 'moon' (litt. “night sun”). See SuN.

55. MOUNTAIN

(1) PAW * $\eta a: k V(\sim g)$ 'mountain' > PW *nuk- > PWN *nok- 'mountain' > Kw. ng-i; PWS *nuč- > Noo. nuč-i: 'mountain' (also Mak. dič-izi:, Dit. duč-i? id.) • PAlg *ma:kw- ( kh)> Yu. mak-w-at 'mountain, peak, hill', mok-w-am- $\partial k^{w}$ 'mountain'.

(2) PNi *chir > NiA., NiS. chir 'mountain'.

(3) PA *wat-y- > WAb. wac-o 'mountain, hill', MiPe. ač-iw-i 'hill, ridge, mountain', FSCr. wač-iy 'hill, mountain'.

(4) Wi. $k^{w}$ as 'hill'. 
56. MOUTH $^{36}$

(1) PAW *m'a:whV $\approx$ *ham'wV 'to eat' (cf. Nikolaev 2015b, \#234) > PNi * $a m-k,-x^{37}>$ NiA. iy- $g$ 'mouth, beak', NiS. am- $x$ 'mouth'. See EAT.

(2) PWN *sams- 'mouth' > KW., Hai. sms, Oo. smms, Hei. smis.

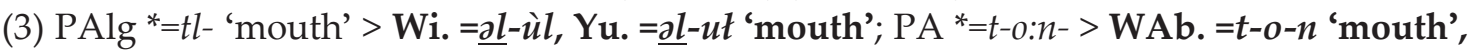
MiPe. $=t-u:-n-i$ 'mouth, beak', FSCr. $o=t-o:-n$ 'her/his mouth'.

57. NAME

(1) PAW *2VklV 'to name' (Nikolaev 2015b, \#42) > PW *-kt- * *kx- 'name(d)' > PWN *-(x)ג-a: 'named, called'> KW. $-(x) \chi(-a)$, Oo. - X(-a), Hei. $-(x) \chi(-a)$, Hai. $-\chi(-a)$; PWS * $-q t->$ Noo. $=q \not(-a)$ 'call, name(d)' (also Mak. $-(k) t-a(:)$ 'having as name', $\{u q u-q t(-a)$ 'one's name is ...', Dit. $-k^{w} a-q t$ 'call, name(d)') $\bullet \mathrm{PNi}{ }^{*} q h a>\mathrm{NiA} ., \mathrm{NiS}$. qha 'name'.

(2) PAlg *w-, "eww, * $w(-e \gamma-)-e n$ - 'to name', * $=w$ - 'name' $38>$ Wi. $w$-an- 'to mention by name', Yu. w-ey-en-ek' 'I call, name' $\mathbf{Y u}$. =ew 'name (n.)'; PA *wi:-n- 'to name' > WAb. wi-s-w-õ$k a n$, MiPe. =wi:-n-s-o:-n-i 'name'. FSCr. $w i:-n-e: w$ TA 'to name so.'.

58. NECK ${ }^{39}$

(1) PAW *q'wi:yV 'neck' (Nikolaev 2015b, \#333) > PWN * $q^{\prime \prime} u$ :- 'neck' > Kw. q'uq'u-n'i, Oo. $q^{\prime w} u q^{\prime w}-n_{0}^{\prime} i$, Hei. $q^{\prime w} u q^{\prime w}-u n_{0}^{\prime} i$, Hai. $q^{\prime} u q^{\prime w}-n i \cdot$ PA * $=k w e: y-a w->$ FSCr. mi-kway-aw 'neck'.

(2) PAW ${ }^{*} c^{\prime} i k^{\prime w} V(\sim \ddot{)})$ neck' > PWS * $c^{\prime} i k^{\prime w}-a m(a) c>$ Noo. c'ik'-umc 'neck' (also Mak. c'ik'w-a:bas,

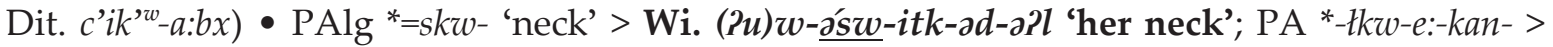
MiPe. $=h k w-e:-k a n-i$ 'neck'.

(3) PAW * $k^{\text {ww }}$ onsV 'neck' (cf. Nikolaev 2015b, \#187) ${ }^{40}>$ PNi *qhos > NiA. qhos ( $\chi$-), NiS. qhos 'neck'.

(4) Yu. pah, paht-un 'neck'.

59. $\mathrm{NEW}^{41}$

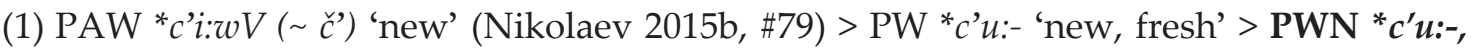
*c'a- 'new'> Kw. cx-as 'new (mat or blanket)'; Oo. c'u-ta, Hai. c'ù-ta 'fresh (said of food)'; PWS ${ }^{*} c^{\prime} u$-š- > Noo. c'u-š.-uk 'new' (also Mak. c'u-s-tk-, Dit. c'u-s-tuk id.) • PNi *chju-řr-> NiA. chu-z-, NiS. chi-r-d 'new' • PAlg *ci:-, *ci- ( ch, č, čh $)>$ Yu. ci-Pn, ca-Pan 'new'.

(2) PAlg *we:ร̌g-, *wě̌g- 'new, young' $42>$ PA *we:šk-, *wešk- > WAb. wsskk-i 'new, young, raw, fresh'; MiPe. we:hk-i- 'new, young'; FSCr. ošk-i 'new'.

60. NiGHT

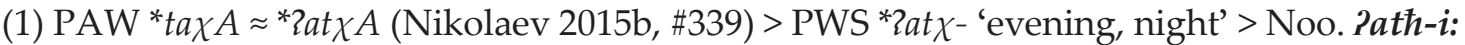

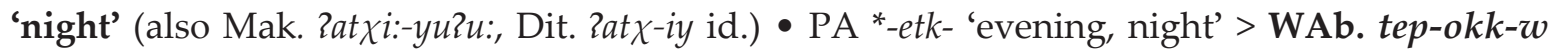
'dark time of the night (later than evening)'; MiPe. tip-ehk-i 'night'; FSCr. tip-isk-a:w II 'to be night, be dark' ("tep- 'dark').

${ }^{36}$ The Noo. data are lacking.

${ }^{37} \mathrm{~A}$ deverbative with the agentive suffix $-k /-x$, cf PNi *hil- $k$, $-x$ 'tongue' from *hel-[h]el- 'to lick'.

${ }^{38}$ The PAW root PNA * $x^{* w} a \approx{ }^{*} h a x^{w w} V$ 'name' (Nikolaev 2015b, \#389) does not really exist.

${ }^{39}$ No data for WAb.

${ }^{40}$ Some of the reflexes of the newly reconstructed PAW root ${ }^{*}{ }^{\prime} i k^{\prime w} V(\sim \ddot{)})$ 'neck' were previously mixed with reflexes of the PAW root ${ }^{*} k^{\text {ww }}$ ons $V$ 'neck' (the second form ${ }^{*}$ ?onsk ${ }^{\text {ww }} A$ is erroneous).

${ }^{41}$ No data for Wiyot.

${ }^{42}$ Also Yu. waz-ay-ay- 'young'. 


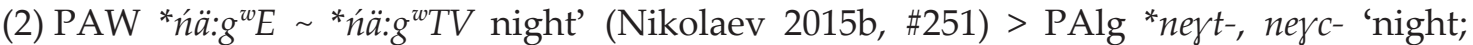
dark' ${ }^{43}>$ Wi. dac-aw-; Yu. nahsc-ew-en 'night'.

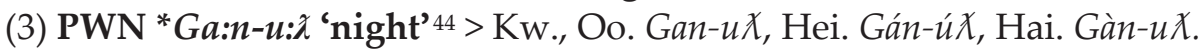

(4) $\mathrm{PNi}{ }^{*}$ wirk > NiA. urk, NiS. irk 'night'.

61. NOSE

(1) PAW *we: $q^{w} E\left(\sim q^{w}\right)$ 'nose, cape' (Nikolaev 2015b, \#373) > PNi *wix > NiA. vix, NiS. ux 'nose'.

(2) $\mathrm{PAW}{ }^{*} q^{w} e s V \approx{ }^{*} h e q^{w} s V$ 'mouth, nose, throat' (Nikolaev 2015b, \#326) > PA *=kwet- 'nose' $>$ FSCr. $o=k o t$ ' his/her nose, its beak'.

(3) PWN * $x^{w} a m$ - 'nose'> Oo. $x^{w} m$-aq, Hei. $x^{w} m$-áq, Hai. xum-àq.

(4) PWS *nic- > Noo. nic-'a 'nose' (also Dit. dic-' id.).

(5) $\mathrm{PA}{ }^{*}=\check{c} y a: t-$ 'nose' > WAb. $=c \tilde{o} l$ 'nose, nostril'.

(6) PA *=kki:w-an-> MiPe. =hkiw-an-i 'nose, bill (of a bird)'.

(7) Wi. tath-ad 'nose'.

(8) Yu. $=\partial p^{\prime}-\partial ? n$ 'nose'.

62. NOT 45

(1) PAW *k'ä: 'negative stem' (Nikolaev 2015b, \#168) > PW ${ }^{*} k\left({ }^{w}\right){ }^{*} i-,{ }^{*}-i k\left({ }^{w}\right)$ ' 'not' > PWN * $k^{\prime}-i$ :-, * $k^{\prime}-u$ :- 'no, not' > Kw. $k$ '- $i$ 'no', $k$ '- $i-w$ - 'no, not, none, nil, non-existent'; Oo. $k$ ' $i$ : 'no', $k^{\prime}-i-w$ - 'not'; Hei. $k$ '-i-á 'no', $k$ '-u-w- 'not', Hai. k'-u 'no', k'-u-s 'not the case, non-existent'; PWS ${ }^{*}-k^{w}>$ Noo. $h i-k$, wi-k 'not' $\bullet$ PAlg ${ }^{*} k a-,{ }^{*} k e-$ 'no, not (negative stem)' > Wi. $k \boldsymbol{d}-$, $k a-$ 'no, not (negative preverb)'; Yu. $k e-, k i-, k o-$ 'no, not (negative stem)'.

(2) PA *mo:- 'negative stem' > MiPe. mu:-hči 'no, not'; FSCr. mo-wa:č 'no, not', mo:-ńa 'no, not (negative marker for independent verbs)'.

(3) WAb. õta 'no, not'.

63. ONE

(1) PAW *n'ə 'one' (Nikolaev 2015b, \#257) > PW *n'a-m > PWN *n'a- $\boldsymbol{m}$-, * $\boldsymbol{m}^{\prime} \boldsymbol{x}-\boldsymbol{n}$ - 'one; alone' $>$ Kw. n'-m-, Oo., Hei., Hai. m'-n- • PNi *ń(i)- > NiA., NiS. ń(i)- 'one' • PAlg *ne-kwet-, "ne-kwec'one' ${ }^{46}>$ Wi. $k u($ ?) $c$, Yu. koht/c- 'one'; PA *ne-kwetw-> WAb. ne-kwec-i; MiPe. n-kot-i 'one'.

(2) PWS *c'a- 'one' > Noo. c'a-w-a:(k) 'one' (also Mak. c'a-w-a:-, c'a-w-i:-, c'a-w-u:-, c'a-k-w$a: 2 a k^{w}$, Dit. c'a-w-a:2k id.).

(3 ) PAlg *pe:r-, *pe:l-> PA *pe:š-47, *pe:l- > FSCr. pe:y-ak'one’.

\section{PERSON}

(1) PAW ${ }^{*} b e: k^{w} E \sim{ }^{*} p e: g^{w} E$ (Nikolaev 2015b, \#62) > PNA *n'a:-be:kw $E \sim{ }^{*} n^{\prime} a:-p e: g^{w} E$ 'person'

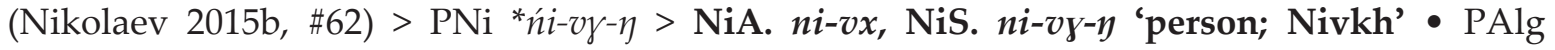
*na:-pe:yw-, "ne-pe:yw-> Yu. pey-ak'man, mal, all-grown-up person'48. See MAN.

(2) $\mathrm{PW}$ *-a:s- 'person' > PWN *-q-a:s, *-G-a:s 'person' > Kw. -Gas, Oo., Hei., Hai. -q-as 'person; fellow; PWS *qu:2-as > Noo. qu:?-as 'person, Indian, adult, man of worth' (also Mak. quz-ac-, qu?-as-, qu:2-as 'husky person, man of worth', Dit. qu:2-as 'man, Indian').

(3) PA *elen-y-iw-> FSCr. ińin-iw 'person, Cree person, aboriginal person'. See MAN.

${ }^{43}$ Also PAlg *n-ey-eyt- > PA *ni:2t- > Men. na-ni:2t-ak-en-a:k-w-at 'night is falling'.

${ }^{44}$ Cf. Oo. Gan-iła, Hei. Gán-íła, Hai. Gan-غ̇ła 'to have supper'.

${ }^{45}$ No data for Nivkh.

${ }^{46}$ A compound, cf. PA *kwet-ak- 'other' and PA *na-w-at- 'first'.

${ }^{47}$ The root PAW * $p E: s ̌ V \approx{ }^{*} 2 E: p \check{s} V$ ‘one' (Nikolaev 2015, \#281) does not really exist.

${ }^{48}$ Cf. per-it 'male'. 
(4) Wi. kuPw-il 'man, Indian, person, human, creature, people' (cf. ku?w- 'to live'). See MAN.

(5) WAb. pemõzsowinno 'a living person, a person'.

(6) MiPe. mihtohse:nia 'human, person, Indian'.

65. RAIN

(1) PWN * $y^{\prime} u: g^{w-}$ 'rain; to rain' > Kw. y'úg ${ }^{w}-a$, Hai. $y^{\prime} u g^{w}-\grave{a}$ 'rain'.

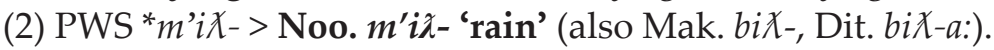

(3) $\mathrm{PNi}$ *lix > NiA. lix 'rain; weather', NiS. lix 'rain'.

(4) PAlg *-phe:?w > Wi. pha?w- 'to rain'; Yu. ten-pew-e?t 'it is raining, rain, storm'.

(5) PA *so:k- 'to pour, soak' > WAb. sok-el-õn II 'it rains'.

(6) PA *kem-iw-an- 'to rain'> FSCr. kim-iw-an II 'to rain'.

(7) PA *pe:t-, *pe:s- 'slowly' > MiPe. pi:t-il-a:n- II 'it rains'.

66. RED

(1) PAW *p'akV ( ä) 'red; blood' (Nikolaev 2015b, \#302) > PNi *pas- > NiA. pas-la-, NiS. pas-d 'red'・PAlg *pak-, "pek- 'to be bloody, red'> Yu. pek-oy-, pak-ay- 'red'. See BLOOD.

(2) PAW *c'ü: $x A \approx{ }^{*}$ ?ü:c'xA 'sap, blood' (Nikolaev 2015b, \#81) PAlg * $=c k-o\left\{w-,{ }^{*}=t k-o\{w-\right.$ 'blood' > PA *me-sk-w- 'red' > WAb. me-kk-w-i; MiPe. me-hk-w- 'red'; FSCr. mi-hk-w-a:w II 'to be red'. See BLOOD.

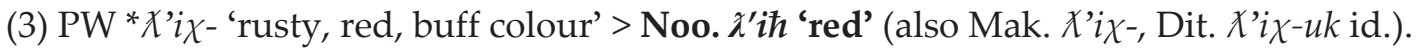

(4) PWN * $\chi^{\prime} a: q$ - 'red'> Kw., Oo. X'aq ${ }^{w}-a$, Hei. $X^{\prime} a q^{w}-a$, Hais. X'aq-.

(5) Wi. $s a y-a ? w$ 'it is red'.

67. ROAD

(1) PNA * $\eta \mathrm{OlyV}(\sim$ ๆ') 'path, road' (Nikolaev 2015b, \#266) > PAlg *mey-e:- > PA *my-e:- > MiPe. mi:-w-i 'road, trail'; PA *mye:-hkanaw- > FSCr. me:-skanaw 'road, path'.

(2) PW *t'ax- 'path, trail, way'> PWN *t'ax- 'pathway, trail, way' > Kw. t'x-la, Oo., Hei. t'x, Hai. t'x-ls; PWS *t'aš- > Noo. t'aš- $i$ : 'road, trail, doorway' (also Mak. t'aš-(i:) 'trail, road, doorway', Dit. t'aš- $i$ : 'trail').

(3) $\mathrm{PNi}^{*} \mathrm{c} i-f>$ NiA. $t i-f(z-, d-)$, NiS. $t i-f$ 'road, path, way, trail'.

(4) Wi. wałal 'road, trail'.

(5) Yu. la: 'road, way'.

(6) WAb. õ wti 'path, trail, road, street'.

68. RoOT

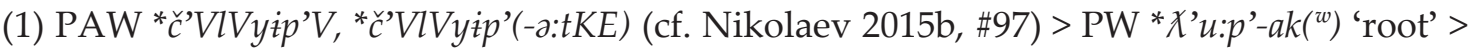
PWN * $\chi^{\prime} u: p^{\prime}-k^{w-}$ 'root' >Kw. X'up'-k, Oo. X'uk'wp, Hei. X'úk'w $p$, Hai. X'up'-k; PWS *X'up'-ač > Noo. $x^{\prime} u p^{\prime}-a c ̌$ root' (also Mak. X'up'-ač id., Dit. X'u:2b-ač 'roots for basket making') • PNi *vizl-ix (metathesis of *zilv-ix) > NiA., NiS vizl-ix $\operatorname{root}^{\prime}$ PAlg *=dlayep-, ${ }^{*}=$ dlayep-i:t(a)k- $(\sim$ th, $k h)$ 'root'>

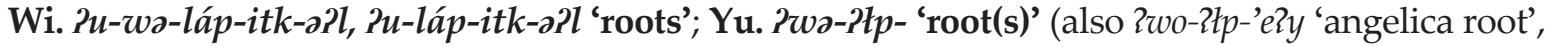
2wa-itp-itak 'root, willow root'); PA *wa=t(y)ap- > FSCr. wa-tap-iy 'root' $\mathbf{\Delta}$ PA *we=tye:p-itk-, *we=tye:p-isk-, ${ }^{*} w e=t y e: p$-išk- 'root' > WAb. wa-capp-kk-w 'plant root'; MiPe. a-či:p-ihk-a 'root (of a plant)'.

69. ROUND

(1) PAW *kOlxV *k'Olk'V * ${ }^{*}{ }^{\prime w} i: l k ' V$ 'round, roundish' (Nikolaev 2015b, \#162) > PWN * $k a l x$ - 'round, circular' > Kw. $k l x$-a 'round, circular, wheel, round thing, etc.', Oo. $k l x$ - $a$ 'circular (an opening)'. 
(2) PNA *2a:wV ( w') 'egg, brood' (Nikolaev 2015b \#21) > PA *w=a:w- 'egg; round' > MiPe. $w$-a:w-i- 'round'; FSCr. $w$-a:w-iy- 'to be round, circular, disk-like'. See EGG.

(3) PNi *pulk- > NiA. pulk-u-, NiS. pulk-u-d 'round'.

(4) PA * pet-ek- 'behind; around' > WAb. pet-ek-w- 'round'.

(5) Noo. cax 'round'.

(6) Wi. Piw- 'to be round'.

(7) Yu. yəhp-əh 'to be round' (cf. Yu. yohp- 'in a circle').

70. SAND

(1) PAW *q'OmbV (Nikolaev 2015b, \#320) > PW * $q$ 'up- 'sand' > PWN * $q$ 'ap- 'sand' > Kw. $q^{\prime} b-g^{w}$ is 'sandy beach', q'b-ilis 'broken shells', Hai. q'p:q'b-is 'sand'; PWS * $q^{\prime} u p->$ Noo.

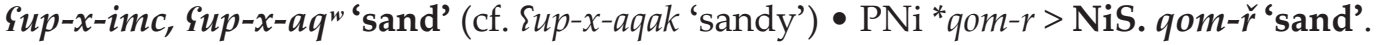

(2) PNA * $p V l-\partial \eta V-k^{\prime w} E$ 'ashes' (Nikolaev 2015b, \#300) > PA *penkw- 'ashes, dust, powder' > WAb. pekw-i 'sand, dust, soil, earth'. See ASHES, EARTH.

(3) PAlg *le:k-, *lek- 'sand' > Wi. latkak 'sand' (perhaps < *lak-ətk 'sandy place', cf. Yu. ri:k-'ew 'shore, sandbar'); PA *le:k-aw-> MiPe. ne:k-aw-i 'sand'; FSCr. nee:k-aw 'sand, fine gravel'.

(4) $\mathrm{PNi}^{*} \operatorname{ma\chi }(\sim \partial)>\mathrm{NiA}$. max'sand'.

(5) Yu. ca-:l (ca?-atk-, ca-: $t k-)$ 'sand, beach'.

71. SAY

(1) PNA *di $\approx{ }^{*} 2 i d V$ 'to say, tell' (Nikolaev 2015b, \#100) > PNi *it- > NiA. it-, NiS. it-t 'to say, tell, speak'.

(2) PW *wa:- 'to say' > Noo. wa:(-t)-, wa-wa: 'to say, speak' (also Mak. wa:(-t)-, Dit. wa:'to say').

(3) PAlg. *he-, *h- 'to say' > Wi. 2-, Yu. $h$ - 'to say'; PA * $\varnothing->$ WAb. it- $\varnothing-a$ TI 'to say', FSCr. it-Ø-e:w TA 'to say to so.', it- Ø-w-e:w AI 'to say', MiPe. $i-\varnothing$ - 'to say'.

(4) PWN *n'i:k- 'to say, tell'> Kw., Oo., Hai. n’ik id., Hei. n’x:n'-ká 'to say repeatedly'.

72. SEE

(1) PAW *n'e: $(w V)$ 'to see, look' (Nikolaev 2015b, \#256) > PW *na:- 'to see, look' > Noo.

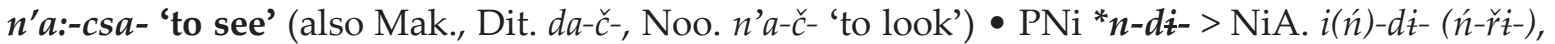
NiS. $i(n)-d \dot{i}-d(n-\check{r} \dot{i}-d)$ 'to see; find' ${ }^{49} \bullet$ PAlg ${ }^{*} n e:(w)-$ 'to see' > Yu. ne-w- 'to see'; PA *ne:- > MiPe. ne:- TA 'to see'. See EYE.

(2) PAlg *wel- 'to see, choose' $50>$ Yu. wal- 'to see'.

(3) PWN *du: $q^{w_{-}}$' to see, look'> Kw. $d u q^{w}-l a$, Oo. duq $q^{w}-l a$, Hei. dú $q^{w}-l a ́$, Hai. dùq ${ }^{w}-l a$.

(4) PA *wa:p-, -[w]a:p- 'white, light; eye, look; to look, see' > WAb. pas-õ $p$ - $i$ 'to see, be able to see'; FSCr. wa:p- 'to see'. See WHITE.

73. SEED ${ }^{51}$

(1) PAW * $t V q{ }^{\prime}\left({ }^{w}\right) E \approx * 2 V t q$ ' $\left({ }^{w}\right) E$ 'bone, gristle' (Nikolaev 2015b, \#213) > PA *we=tk-an- 'bone;

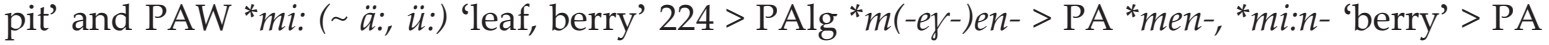
*wełkan-i-men- 'seed, pit' > WAb. sskkan-i-men 'seed'; MiPe. ahkan-i-min-i 'seed (of a fruit, in the middle)'; FSCr. ohka-ta:-min 'seed, nut, pit'. See BONE.

(2) PNi *xem > NiA., NiS. xem 'seed, grain'.

${ }^{49} \mathrm{Cf}$. the suffixed forms PNi *n- $u$ - 'to look, watch', *na- $\chi$ 'eye'; PA *na-t- 'to seek, hunt'.

${ }^{50} \mathrm{PA}$ *wel- 'to choose'.

${ }^{51}$ No data for PWN, Noo., Wi., or Yu. 
74. SIT

(1) PAW ${ }^{*} t^{\prime} i: q^{\prime w} V \sim{ }^{*} t i: q^{\text {ww }} V$ 'to sit' (Nikolaev 2015b, \#350) > PNi *thiv- > NiA. iřp- (thiv-, riv-), NiS. iřp-t (thiv-) 'to sit; sit down'.

(2) $\mathrm{PW}{ }^{*} k^{\prime w} a:-,{ }^{*} k^{w} a:->\mathrm{PWN}{ }^{*} k^{\prime} w a:-$ 'to sit' > Kw. $k^{\prime w} a-t a$ 'sitting, to be seated, meeting', Oo. $k^{\prime w} a$ :-la 'sitting, a marriage ceremony', Hei. $k^{\prime w} a$-la 'sitting, a marriage ceremony', Hai. $k$ wà:-la 'sitting'; PWS ${ }^{*} k^{w} a->$ Noo. $k^{w} a$ - 'to sit' (also Mak. $k^{w} a$ - id., Dit. $k^{w} a$ - 'to sit down').

(3) PAlg *-ap?- 'to sit', *-i:pz- 'to put' $52>$ PA *-ap-, *-ep- > WAb. l-ap-i 'to sit'; MiPe. ap-iAI 'to be located, sit'; FSCr. $a p-i$ - 'to sit, stay in a place'.

(4) Wi. tam- 'to sit'.

(5) Yu. rek'i:n 'to sit'.

75. SKIN 53

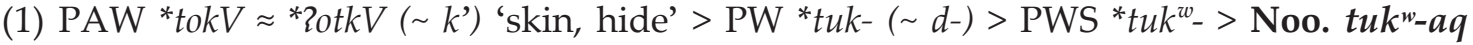
'skin, hide' (also Mak. tukw-aq id.) - PAlg *=tak-, =atk-, =ack- 'skin' > Wi. w-otk-ay 'skin'; Yu. $2 w$-əs, ?w-əsk-un 'skin'; PA *=tak-> WAb. ma-tak-en 'hide, skin'.

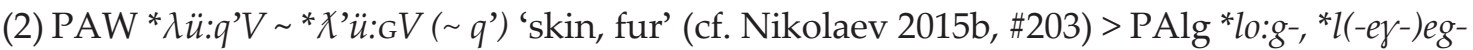
'skin, feather' $54>$ PA *-lo:k- ( t) 'hide, skin' > MiPe. =lu:k-ay-i 'skin'. See FEATHER.

(3) PNA *2V $r r V$ 'skin (of animals), scale' (Nikolaev 2015b, \#41) > PAlg * $=a \gamma l-a k-,{ }^{*}=e \gamma l-e k-$, *a $a r r-a k$ - 'skin, shell' 55; PA *-ąl-ak-, *-ęš-ak- 'skin, scale' > FSCr. $m=i \check{s}$ - $a k$-ay 'skin (animal, person, tree)'.

(4) $\mathrm{PW} * \chi ' i$ :- 'skin (of fish, animal, human)'> PWN * $\chi^{\prime} i$ :-s- 'skin (of fish, animal, human, fruit)'> Kw. , Hei X'i-s, Oo. X'i:-s, X'is, Hai. X'i:-s.

(5) $\mathrm{PNi}$ *hal ( a) ${ }^{56}>\mathrm{NiA}$. hal 'skin (of human); body'.

76. SLEEP

(1) PAW ${ }^{*} k^{\text {ww }}$ :tV $\approx{ }^{*}$ ?o:tk ${ }^{w} A$ (cf. Nikolaev 2015b, 188) > PWN * $k^{\prime} a: t-$ 'to sleep' > Kw. $k$ 'at-a, Hei. k'át-a, Hai. k'àt-a • PNi *qho- > NiA. qho-, NiS. qho-d 'to sleep' • PAlg *-i:tkw- 'to sleep, dream' > Wi. $\boldsymbol{n}$-itw- 'to sleep' ${ }^{57}$.

(2) PAW *'n'AbV ( p') 'to die' (Nikolaev 2015b, \#255) > PA *nep- 'to die; sleep' > MiPe. nep-a:- AI 'to sleep'; FSCr. nip-a:w AI 'to sleep, be asleep'.

(3) PWS *warič- > Noo. werič 'to sleep' (also Mak., Dit. werič).

(4) PA *kaw- 'to prostrate' > WAb. kaw-i AI 'to sleep'.

(5) Yu. ckey-ek' 'I sleep, I am asleep'.

77. SMALL

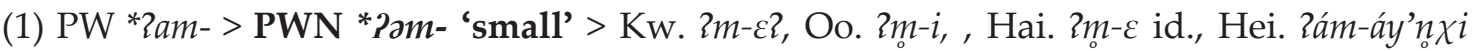
'youngest child in the family'; PWS *2ap-a:s- > Noo. 2ap-a:s- 'small' (also Mak. 2ap-a:s 'nice, cute', Dit. 2ap-a:s 'nice').

(2) $\mathrm{PNi}$ * mac-ki-> NiA. mac-ki-, NiS. mic-ki-d 'small'(cf. *mac-u- 'to diminish').

(3) PA * pi:w- 'small piece' > WAb. piw $i$ 'small, fine, thin'. See THIN.

${ }^{52}$ Also Yu. -ip-'- in skew-ip'-ak' 'I put in order', etc.

${ }^{53}$ No data for NiS.

${ }^{54}$ Also Yu. re?- $n$-oh 'feather', $r$-ej-o? 'feather, feather for display (as in headband), morning feathers (in brush dance)'.

${ }^{55}$ Also Yu. sl-ek-w 'clothes [a single set]', etc.

${ }^{56} \mathrm{PNi}{ }^{*} h-<* q h-$, cf. the Yukaghir borrowing * qhal- 'bark, scales'.

${ }^{57}$ Also Yu. -it 'sleep, dream'. 
(4) PA *apeHt-, *apeȞ̌- > MiPe. apihš- 'narrow, small'; FSCr. apihč-i 'small, little, midget'.

(5) Wi. bokt'small'.

(6) Yu. ceyk- 'small; narrow (flat things)'.

78. SMOKE 58

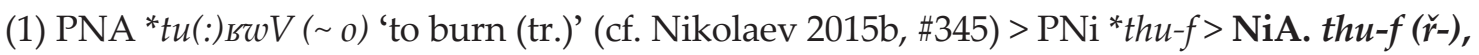
NiS. thu-f'smoke'. See BURN, FIRE.

(2) $\mathrm{PW}{ }^{*} q^{w} a: y x$ - 'smoke, to smoke' > PWN ${ }^{*} \boldsymbol{k}^{w} a: x$ - 'to smoke (said of fire or chimney)' >

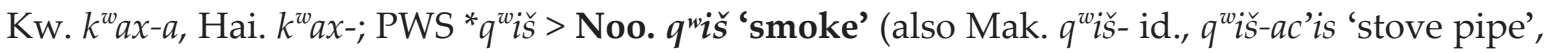
Dit. q'iš-a: 'smoke').

(3) WAb. peketa 'smoke'.

(4) MiPe. a:hkoli 'smoke' (cf. PA *či:p-a:hkww- 'to cook).

(5) Wi. birwad 'smoke'.

(6) Yu mera: 'smoke'.

79. STAND

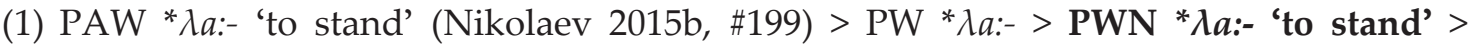
Kw. $\lambda a-\left\{a\right.$ 'standing, to be upright', $\lambda a-\chi^{w}$ - 'to stand (largely, but not entirely, limited to humans), upright', Hei. $\lambda a{ }^{-} \chi^{w}-$ lít 'to get up, get out of bed, to volunteer', Hai. $\lambda \grave{a}-\chi^{w}$-lit 'to get up,

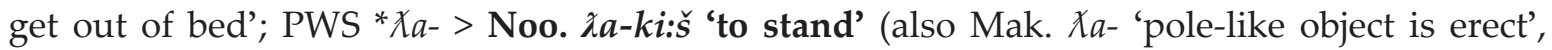
* Xa-ki(:)̌s- 'to stand (human)', Dit. Xa- 'stick-like object stands up', Xa-kiš(šX)- to stand').

(2) PNA * ga:p’ $V$ 'to stand' (Nikolaev 2015b, \#111) > PNi *kap-r-> NiA. kip-r-, NiS. kap-r-d 'to stand; stand up' • PAlg *ga:p- $(\sim$ ph) 'to stand' > Yu. -o-Pop 'to stand'; PA *-ka:p-, *kap- > WAb. $k \tilde{o} p$-o AI 'he stands'.

(3) PA *ni:p- 'to stand' > MiPe. ni:p-aw-, FSCr. ni:p-aw- 'to stand'.

(4) Wi. $-a$ ?-w-, $-a$ ?-y- 'to stand'.

80. STAR ${ }^{59}$

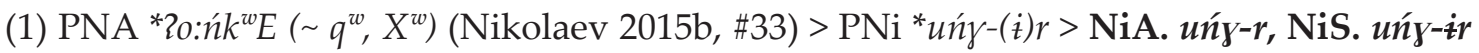
'star' • PA -a:nkw- 'star' > WAb. al-akkw-ss; MiPe. al-a:nkw-a; FSCr. wač-ahk-oš 'star'.

(2) PW *t'a:w- > PWN *t'u:- 'star' > Kw. t'ut'u, Oo. t'ut'u-a, Hei. t'út'u-za id.r', Hai. t'ut'u-za 'North Star'; PWS *t'a:w- > Noo. t'at'u(:)-s 'star' (also Dit. t'a:t'aw-ąsi? id., Mak. t'a:w-isa:bac 'stars').

(3) Yu. ho:y-ec 'star'.

81. STONE

(1) PNA * piLV-lVk'(w)E ( a) 'stone suitable for making tools' (Nikolaev 2015b, \#287) > PAlg *peletk-60 > Wi. płatk 'rock, stone'.

(2) PW *t'i:- 'stone weight' > PWN *t'i:-s- 'stone'61 > Kw. t'i-s-ma 'stone, rock, ore', Oo. $t$ ' $i-s-a$ 'to weight with a stone', Hei. $t$ ' $i$-s-mo, Hai. $t$ 'ì-s-m 'stone, rock, ore'; PWS *t' $i$ - > Noo. $t$ ' $i$ '(big) stone' (also Mak. t'i-di:č 'rock' 62, Dit. t'i-dičk ${ }^{w}$ 'stone, rock').

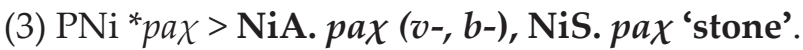

\footnotetext{
${ }^{58}$ No data for FSCr.

${ }^{59}$ No data for Wiyot.

${ }^{60}$ Also Yu. petk-ot 'pebbles, gravel'; PA *-a:-petkw-, *-pełkw- 'stone; metal'.

${ }^{61} \mathrm{Cf}$. PWN *t'i:- 'dead weight, ballast'.

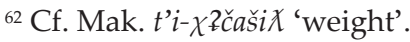


(4) PA *a?s-en- 'stone, rock' > WAb. ass-en 'stone'; MiPe. ahs-en-i 'rock, stone'; FSCr. as-in-iy 'stone, rock'.

(5) Yu. ha?a:y 'rock'.

82. SUN ${ }^{63}$

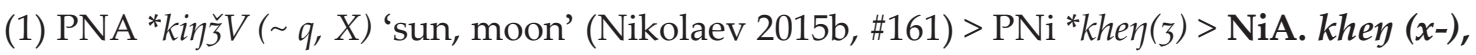
NiS. khey 'sun' - PAlg *k-ej-ečh- 'sun, moon' > PA *ki:š- 'sun, moon, month; day, sky' > WAb. kis-oss 'sun, moon, luminary'; MiPe. ki:t-s-w-a 'sun, moon; month'. See MoON.

(4) FSCr. pi:sim 'sun; month'. See MOON.

(2) Noo. hupat'soon, moon, month'. See MoON.

(3) Yu. won=erwsley 'moon, sun' (won- 'up'). See MOON.

83. SWIM

(1) PAW *ma:rV (Nikolaev 2015b, \#222) > PWN *ma:t- 'to swim' > Oo. mat-la, Hei. mát-, Hai. màt-la • PNi *mra- > NiA. mri-, NiS. mra-d 'to swim (human, animal), bathe' • PAlg *-[m]o:l- > Wi. -ul-; Yu. -ur- 'to swim'.

(2) PWS *sus- > Noo. sus 'to swim' (also Mak. sus-, Dit. sus-a:).

(3) PA *a:tak- 'swim' > FSCr. pim-a:tak-a:w AI 'to swim; to wade'.

(4) WAb. takkassmi AI 'to swim'.

(5) MiPe. -i:čime: AI 'to swim'.

84. TAIL

(1) $\mathrm{PAW}{ }^{*} h V_{3} V \approx * 2 h_{3} V$ 'tail (of quadruped)' > PWN *has- $(\sim c)$ 'tail (of animal) $>$ Oo., Hei. hc-' $\chi$ dí id., Kw. hc-' $\chi$ sdi? 'tail of an animal or fish' $\bullet$ PAlg ${ }^{*}=e c h-,{ }^{*}=e t h$ - 'tail of quadruped' $>$ Wi. $w$-oth- 'tail'; PAl ${ }^{*}=s-0: w-,=s-w$ - 'tail (of quadruped)' $>$ MiPe. $=n-s u$ : $-y$ - $i$ 'animal's tail (not a bird's tail)'; FSCr. $o=s w$-ay 'tail'.

(2) PAW * $\eta^{\prime} \partial: g E\left(\sim k^{\prime}\right)$ 'tail' (Nikolaev 2015b, \#270) > PNi * $y \jmath k-i>$ NiA. yək-i, NiS. yak-i 'tail'.

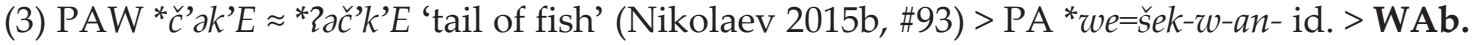
o-sok-en-a 'tail'.

(4) PAW *či:t’V(-lV) 'foot, leg, flipper' (Nikolaev 2015b, \#86) > PWS *sit- > Noo. sit-'a 'tail'. See Foot.

(5) PAlg * =at- 'bird's tail' ${ }^{4}>$ Yu. $=a t-a y$ 'tail'.

85. THAT 65

(1) PAW * $y V$ - 'demonstrative stem' (Nikolaev 2015b, \#412) > PWN *ya:- 'that' > Kw $y-\chi-(a)$ 'that', Hei. yá 'that over there', Hei. ya- $\chi$ - $t$ 'thus'; PWS *ya:- > Noo. ya: 'that, there' (also Dit. ya: 'that', Mak. ya:t, ya(:)-t 'there').

(2) PAW * $2 V$ - 'demonstrative stem' > PNi ${ }^{*} a->$ NiA. $a-z$, NiS. $a-u-d, a ́-h u-d$ 'that (distant, but visible)'.

(3) PAW ${ }^{*} g V \sim{ }^{*} g^{w} V$ 'demonstrative stem' (Nikolaev 2015b, \#108) > PAlg *kV- > Wi. $\boldsymbol{k}$ - $\boldsymbol{u}$-; Yu. $\boldsymbol{k}$ - $\boldsymbol{u}$ - 'that'. See THIS.

(4) PA *an-, ${ }^{*}-e n->$ WAb. $n-a$ (anim.), $n-i$ (inanim.); MiPe. i:n-a:n-a (anim.), $i: n-i: n-i$ (inanim.); FSCr. an-a (anim.), an-i-ma (inanim.) 'that' 66 .

${ }^{63}$ No data for PWN and Wiyot.

${ }^{64} \mathrm{PA} * w=a t-a n-y$ - 'bird's tail'.

${ }^{65}$ No data for PWN.

${ }_{66}$ This form also contains the PA morpheme * $m V-$, see THIS. 
86. THIS

(1) PAW ${ }^{*} g V \sim{ }^{*} g^{w} V$ 'demonstrative stem' (Nikolaev 2015b, \#108) > PWN * $g a$ :- 'this (near speaker)' > Kw., Hai. ga, Oo. ga, ga:, Hei. gá • PAlg *kV-> Yu. $k$-' $\boldsymbol{i}$ - 'this'. See THAT.

(2) PAW * $d V$ 'demonstrative stem' (Nikolaev 2015b, \#107) > PNi ${ }^{*} t$-wi- $>$ NiA. $t \mathbf{i}-\mathbf{z}$, NiS. $t u-d$ 'this'.

(3) PAW *y'V 'demonstrative stem' (Nikolaev 2015b, \#412) > PA *yV- > WAb. yo 'this (inanim.)'. See THAT.

(4) PAW * $w$ 'V 'demonstrative stem' (Nikolaev 2015b, \#386) > PAlg * $w V$ - > Wi. $w$ - $u$ - 'this'; PA * $w V->$ WAb. $w$ - $a$ 'this (anim.)'; FSCr. $a-w-a$ (anim.) 'this'.

(5) $\mathrm{PW}^{*} \chi\left({ }^{w}\right) V-,{ }^{*}-\chi\left({ }^{w}\right)-\left(\right.$ cf. PNW ${ }^{*} \chi \chi^{w} a-$ 'this [near you]') $>$ PWS ${ }^{*} 2 a-\chi>$ Noo. $2 a-h$ 'this'.

(6) PA *an-, *-en-> MiPe. o:n-a:n-a (anim.), o:n-i:n-i (inanim.) 'this'. See THAT.

(7) PA *-mV> FSCr. o:-ma (inanim.) 'this'. See THAT.

87. THOU

(1) PAW * $k V$ 'you (sg.), thou' (Nikolaev 2015b, \#164) > PNi *chi > NiA., NiS. chi 'you sg.' • PAlg ${ }^{*} k e$ - (pref.) 'thou, thee, thy', ${ }^{*} k e-$ - $i l-a$ 'thou' > Wi. $k$-hil; Yu. $k$-'el 'you sg.'; PA *k-i:l-a > WAb. $k$-i-a; MiPe. $k$-i:l-a; FSCr. $k-i: n-a$ 'you sg.'.

(2) PW *su:-, *-u:s- > PWN *su:-, suff. *-u:s 'you sg.' > Kw. suff. -(u)s 'you', sù- $2 m$

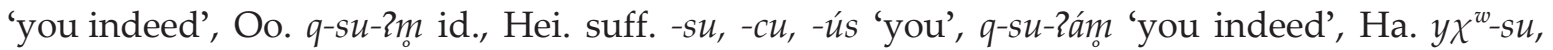
suff. $-s u,-s$ 'you'; PWS *su(:)-, *-us- > Noo. su-w'a 'you sg.' (also Mak. su-wa:, pref. sut-, suff. -su:, Dit. $s u-w$ 'a (indep.), suff. $-s u-k^{w},-2 a s,-c u-\chi$ 'you sg.').

88. TONGUE

(1) PAW *hi:tV ( e:) 'tongue; to lick' (Nikolaev 2015b, \#148) > PNi *hil-k, $-x>$ NiA. hil- $x$, NiS. hil-k 'tongue' (cf. *hel-[h]el- 'to lick') - PA * ${ }^{*}=e: t-a l->$ WAb. =il-al-o 'tongue', FSCr. ot-e:ń-ań-iy 'her/his tongue'.

(2) PNA*iz:płV 'lip, tip of tongue' (Nikolaev 2015b, \#31) > PAlg *i:pt- > Wi. =it, Yu. =ipt 'tongue'; PA * =i:t-an-> MiPe. $=i: l-a n-i$ 'tongue'.

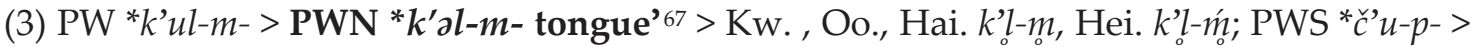
Noo. $\check{c}$ 'u-p 'tongue'.

89. ТОотн

(1) PAW * Gi:gE 'tooth, fang' (Nikolaev 2015b, \#123) > PW *gi:k- > PWN *gi:k- 'tooth, teeth' $>$ Kw. gigi, Oo., Hei. gik, Hai. gig; PWS *kik-> Noo. čičič- $i$ 'tooth' (also Mak. čič-, Dit. čič-iz(i:)).

(2) PAW * $x E c V \approx{ }^{*} 2 E x c V(\sim s)$ 'tooth' (Nikolaev 2015b, \#388) > PNi ${ }^{*} \eta=\partial \gamma s>$ NiA. $\boldsymbol{y}$-i $y s$, NiS. $y$-ays, $y$-ayz-iř 'tooth'.

(3) PAlg ${ }^{*}=(e y-) e p-e t-68>$ Wi. $=\partial p-t$ 'tooth'; PA * $=i: p-e t->$ WAb. $=i p-i t$, MiPe. $=i: p-i t-i$, FSCr. $w$-i:p-it 'tooth'. See BITE, EAT.

(4) Yu. =arpet 'tooth, teeth'.

90. TREE $^{69}$

(1) PAW ${ }^{*} i^{2}{ }^{w} E\left(\sim k^{w w}\right)$ 'tree' (cf. Nikolaev 2015b, \#416) > PW *suk- > PWS *suč- > Noo. suč-'as

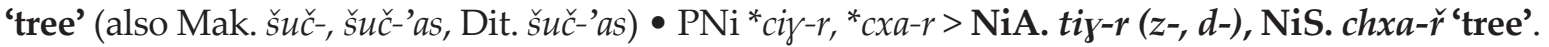

(2) PAlg *-a:2-, -i:2-> Wi. =át-i? 'wood, stick, tree'; Yu. tep-o: 'tree' (with suff. -o? 'tree, stem').

${ }^{67} \mathrm{PWN} * k$ 'al- 'to lick', cf. PWN *k'al-q- 'to lick'.

${ }^{68}$ Cf. PAlg *-(2)ep- 'by tooth, to bite, eat'.

${ }^{69}$ No data for PWN. 
(3) $\mathrm{PA}^{*}=(e)$ it-ekw- > FSCr. $m i$-st-ik 'tree'.

(4) PA *apanš- 'tent-pole; squared timber, rafter' > WAb. apas- $i$ 'tree, a woody plant large enough to contain firewood'.

(5) PA *aht- 'tree ?' ${ }^{70}>$ MiPe. aht-aw-a:n-i 'tree, wood; stick'.

91. TWO

(1) PAW *me: 'two' (Nikolaev 2015b, \#219) > PW *ma:-71 > PWN *ma:-2t 'two' > Kw., Oo., Hei. ma-2t-, Hai. ma-t-' $\bullet \mathrm{PNi}^{*} m i$, “me > NiA., NiS. mi-, me- 'two'.

(2) PAW *n't- 'two' (cf. Nikolaev 2015b, \#408) > PAlg ${ }^{*} n i-\check{z}-,{ }^{*} n i-d-,{ }^{*} n-e \gamma-i-\check{z}->$ Wi. di-t, Yu. ni-?- (also no-?-, nə-?-) 'two'; PA *nyi:-š- > WAb. ni-s; MiPe. ni:-š-w-i; FSCr. ni:-š-o 'two'.

(3) PWS *2aXa > Noo. ?aגa 'two' (also Mak. ?aX(a), Dit. ?aXa).

92. WALK $(\mathrm{GO})^{72}$

(1) PAW *wi ( e) 'to walk, go' (Nikolaev 2015b, \#375) > PNi *vi- > NiA. vi-, NiS. vi-d 'to walk, go'; PA *we-ht-> WAb. -o-ss-a, -ó-ss-a, -a-ss-a, MiPe. -ohs-e: 'to walk'.

(2) PW *ya:- ${ }^{73}$ 'to move, step' > Noo. ya:-c-uk 'to walk, go, proceed' (also Dit. ya-c'to walk on, go (on), nudge with foot';).

(3) PWN *tu:- 'to walk'> Oo., Hai. tu-a, Hei. tu-á.

(4) Wi. Pal- 'to go, walk'.

(5) Yu. hey- 'to go, walk, travel, dance'.

93. WARM

(1) PAW ${ }^{*} k^{w} \ddot{a}: x^{w} V$ 'warm, hot' (Nikolaev 2015b, \#176) > PWN * $\boldsymbol{k}^{w} u: \boldsymbol{x}^{w_{-}}$'warm, hot' > Oo. $k^{w} u x^{w}-a$, Hei. $k^{w} u x^{w}-$ a, Hai. $k \grave{u} x^{w}-l a$ id., Kw. kux $x^{w}-l a$ 'mild weather'.

(2) PW *X'u:p- 'to heat up' > PWN *X'u:p- > Noo. $\chi^{\prime} u p-a:$ 'warm, hot' (also Mak. X'up-, X'ub-, Dit. X'up-).

(3) $\mathrm{PNi}{ }^{*}$ tok- > NiA. tik-la-, NiS. tak-t 'warm'.

(4) PAlg *k(-ey-)et- 'hot' > PA *keš-y-, *ki:š-y- 'hot, warm' > WAb. kes-ap-es-o AI 'he is warm', kis-op-att-a II 'the water is already warm', etc.; MiPe. kiš-a:p-ihk- 'hot, warm'; FSCr. kis-is-ow- 'to be warm'.

(5) Wi. was- 'warm; to heat'.

(6) Yu. hewom- 'warm'.

94. WATER

(1) PAW *w'e:pV 'water, liquid' 74 (cf. Nikolaev 2015b, \#38475) > PWN *w'a:p- 'water' > Kw., Hai. w'ap 'water', Kw., Oo. w'ap-a, Hei. w'áp-a, Hai. w'àp-la 'to dilute, to water down'.

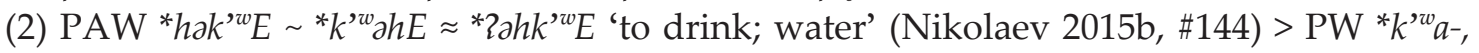
${ }^{*} k$ 'a- > PWS *č'a-76> Noo. č́ $a-? a k$ 'water' (also Mak. ča-2ak', Dit. č'a-2ak 'water').

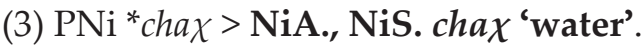

${ }^{70}$ Cf. *aht-a:py- 'bow', "tree ?+string".

${ }^{71} \mathrm{Cf}$. PWN *ma-t- 'twin(s)'.

${ }^{72}$ No data for FSCr.

${ }^{73}$ Also PWN *ya:- > Kw. ya-la 'keep on going'.

${ }^{74}$ The same root in PAlg *-[w]a:p- $(\sim p h)>$ Yu. suff. -op- 'water, liquid'; PA *-[w]a:p- 'liquid, water'.

75 Pace Nikolaev 2015b, \#384, Yu. par-ah actually belongs to a different root - PAlg *nepiz- 'water'; consequently, the root variant PAW * $p e: w^{\prime} V$ has to be eliminated.

${ }^{76}$ Cf. Dit. ča-y'awa: 'always get water'. 
(4) PAlg *nepiz- > Yu. pa?-ah 'water'; PA *nерy- > WAb. nepi 'water, liquid, sap'; MiPe. nipi, FSCr. nipiy 'water'.

(5) Wi. Pu?l 'water'.

95. WE (EXCLUSIVE)

(1) PAW *ńV- 'I; we (excl.)' (cf. Nikolaev 2015b, \#254) > PW *nu:- 'I, we' > PWN *nu:-? $\boldsymbol{k}^{w}$ 'we (excl.)' > Kw. $-n u-\left\{x^{w}\right.$, Oo. $-n u-k^{w}$, Hei. $-n-t k^{w}$, Hai. $-n u-k^{w}$; PWS *nu:-, *ni:- > Noo. ni:- $w^{\prime} a$

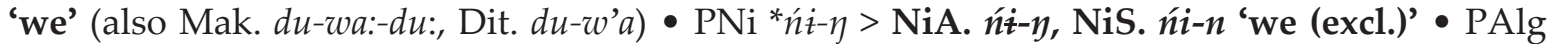
*ne- 'we (excl.) > Wi. Pi-nà-d, Yu. ne-k-ah 'we'; PAlg *n-i:l- > WAb. ni-on-a, MiPe. n-i:l-u:n-a, FSCr. $n$-i:ń-an-a:n 'we (excl.)'. See I.

96. WHAT

(1) PAW ${ }^{*} q V(\sim \mathrm{G})$ 'interrogative stem' $>\mathrm{PW}{ }^{*} q V-(\sim \mathrm{G})>$ Noo. $? a-q-i-, p a-q-a q$ 'what' • PAlg *ke:- > PA *ke:-kw- > WAb. $k a-k w-i, k a-k w-e s s-a$ MiPe. $k e$ :-tw-i; FSCr. ke:-kw-a:n 'what?'.

(2) PAW ${ }^{*} g^{w} V$ 'interrogative stem' (Nikolaev 2015b, \#118) > PAlg ${ }^{*} k w->$ Wi. $k^{w}-a-t-w a$ 'what?'; PA *ke:-kw- > WAb. ka-kw-i, ka-kw-ess-a MiPe. ke:-tw-i; FSCr. ke:-kw-a:n 'what?'. See WHO.

(3) PNA *tV 'interrogative stem' (Nikolaev 2015b, \#346) > PNi *thi-, *thu- > NiA. si-z, NiS. řu-d 'what?' • PAlg *ti:- > Yu. ti(?)- 'what?'.

(4) PW * m'a:- 'interrogative stem' > PWN *m'a:- 'what?' > Kw. m'a, m'a-s, Oo. m'a, m'a:-s, Hei. m'á, m'á-s, Hai. m'a-s.

97. WHITE 77

(1) PW X'i:s- > 'white, white hot' > Noo. X'ic 'white' (also Mak., Dit. X'is-).

(2) PWN * $m^{\prime} u: q^{w_{-}}$'white, discoloured, bland, stale'> Oo. m'uq $q^{w}-a$, Hei. m'ú $q^{w}-a$, Hai. $m$ 'ù $q^{w}-a$ id., Kw. $m$ 'u $u{ }^{w}$-sm ' 'grey-haired'.

(3) $\mathrm{PNi}{ }^{*}$ qhon-u-> NiA. qhon-u-, NiS. qhon-u-d 'white'.

(4) PA *wa:p- 'white, light; eye, look; to look, see' > WAb. wõ p-i, MiPe. wa:p- 'white', FSCr. wa:p-a:w II 'to be white'. See SEE.

(5) Yu. munc- 'white'.

98. WHO

(1) PAW * $g^{w} V$ 'interrogative stem' (Nikolaev 2015b, \#118) > PW *2a(n)-g(w)- > PWN *2ə(n)-gwa:

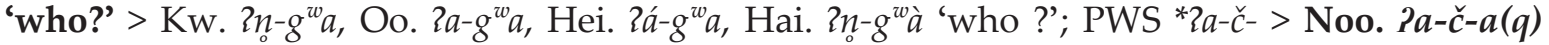
'who?' (also Mak. 2ač-aq, Dit. 2ač-(aq)) • PAlg ${ }^{*} k w$ - $>$ Wi. $\boldsymbol{k}^{w-i}-\mathfrak{t w a}$, Yu. $\boldsymbol{k}$-' $\boldsymbol{i}, \boldsymbol{k}$-u 'who'. See WHAT.

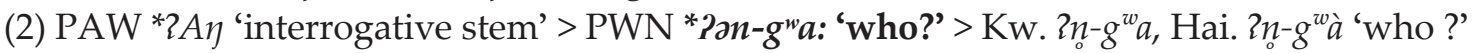
- $\mathrm{PNi}{ }^{*} a \eta>\mathrm{NiA}$. $a y$ 'who, where'.

(3) $\mathrm{PAW}{ }^{*} n a \approx * 2 a n V$ 'interrogative stem' (Nikolaev 2015b, \#241) > PNi *na- ( a) > NiS. $n \boldsymbol{a}-\boldsymbol{r}$ 'who' - PA *-e:n-, -an-> WAb. aw-an-i 'someone; who?', MiPe. aw-e:n- $a$, FSCr. aw-e:n- $a$ 'who'

(4) $\mathrm{PAW}{ }^{*} w V \approx 2 V w V\left(\sim w^{\prime}\right)$ 'interrogative stem' (cf. Nikolaev 2015b, \#381) > PA *aw- > WAb. aw-an-i 'someone; who?', MiPe. aw-e:n-a, FSCr. aw-e:n-a 'who'.

99. WOMAN

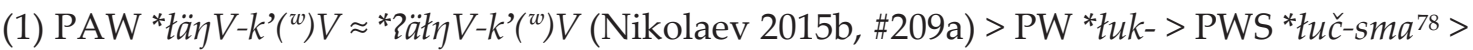

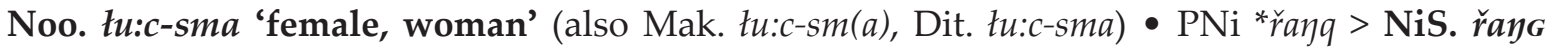
'woman' • PAlg *2ałkw-, *2etkw- ( kh) 'woman, female'> FSCr. iskw-e:w 'woman'.

${ }_{77}$ No data for Wiyot. 
(2) $P W N$ *Gan- 'female, woman, wife, daughter, girl' > Oo. Gn-mo, Hei. Gn-mó, Hai. Gn-mo id., Kw. Gn-m 'wife'.

(3) PNi *umg-u> NiA. umg-u 'woman'.

(4) WAb. pehanem 'woman, wife'.

(5) MiPe. mitemohsa 'woman'.

(6) Wi. kabuč 'woman, women'.

(7) $\mathrm{Yu}$. wencok's 'woman'79.

100. YELLOW ${ }^{80}$

(1) PNi *evrq-> NiA. evrq-, NiS. evřq vala-d 'yellow'.

(2) PA *wes- 'yellow, brown' > WAb. wis-õw-ik-, FSCr. os-a:w- 'yellow'.

(3) MiPe. u:ns-a:w- 'yellow'.

(4) Noo. hicp'iqak 'yellow' (litt. "like excrements").

(5) Yu. ta:nep, tirnp- 'to be yellow'.

101. FAR

(1) PWN * $\chi^{w i}$ :ss-, * $q^{w i}$ :ss- 'that direction, far, on the far side, far away (in time or space)'

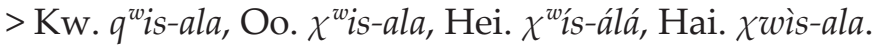

(2) PWS *su:-81 > Noo. $-s u(:)$ 'far'.

(3) PNi *thi-> NiA. thi-la-, NiS. thi-d 'far'.

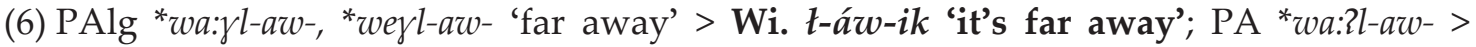
FSCr. wa:ń-aw 'far, distant'.

(7 ) PAlg *no:2-aw-> Yu. nu:?-w 'far away'; PA *na:-w- > WAb. nõ-w-i 'far, long'

(8) PA *pel-aw- 'far' > MiPe. pil-w-i 'far away, far off'.

102. HEAVY

(1) PWN * $G^{w i}$ :- 'weighing heavy' > Oo. $G^{w} i-u k^{w}$, Hei. $G^{w} i-u k^{w}$ id., Hai. $G^{w} i-u g^{w} a s$ 'weight (of a person, etc.)'.

(2) PNi *per-> NiA. per-la-, NiS. per-d 'heavy'.

(3) PA *kwes-ekw- > FSCr. kos-ikw- 'to be heavy'.

(4) Noo. $k^{w}$ atyi:k 'heavy'.

(5) WAb. ttekkw-ikw- 'heavy'.

(6) MiPe. kahč-ok(w)- 'heavy'.

(7) Wi. loy- 'heavy'.

(8) Yu. pke?y- "heavy'.

103. NEAR ${ }^{82}$

(1) PAW *ma: 'near' (Nikolaev 2015b, \#215) > PNi *ma- > NiA. ma-, NiS. ma-d 'near'.

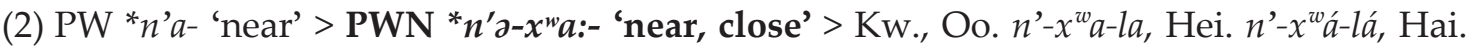
$n$ '- $x^{w}-a \grave{a}$-la; PWS *n'a-> Noo. na-t 'near'.

(3) PAlg *ček-, "t-ej-ek- 'close, near' > Yu. ck-war-ak' 'near'; PA *či:k-> MiPe. či:k-a 'near; almost'.

(4) PA * peHš- 'to touch; near' > WAb. pass-ot-a 'near', FSCr. peš-oč 'close by, near'.

\footnotetext{
${ }^{78}$ Cf. Quil. -siba 'wife'.

${ }^{79}$ Cf. Yu. wentok ${ }^{w}$ ' 'female (animal or bird)'.

${ }^{80}$ No data for PWN and Wiyot.

${ }^{81}$ Cf. Quil. sawa 'to go far'.

${ }^{82}$ No data for Wiyot.
} 
104. SALT(Y)

(1) $\mathrm{PW}{ }^{*} d u m$ - 'salt water' > PWN *dam- 'saltwater, sea' > Kw. $d m-x s$, Oo. $d m-2 x s$, Hei. dm-xs, Hai. dm-ks; Noo tup-'at 'saltwater' (also Mak., Dit. tup-'at).

(2) PNi *hap-> NiA. hap-la-, NiS. hap-t'salty'.

(3) PA *ši:w- 'strong taste (sour/salty)' > WAb. sizw-an, FSCr. ši:w-ah-am 'salt'.

(4) PA *wi:nk- 'sweet, tasty'> MiPe. wi:hk-ap-a:k-an-i 'salt'.

(5) Wi. phákal- 'salty (water)'.

(6) Yu. Pewp-oh 'salt water, Pacific Ocean'.

105. SHORT ${ }^{83}$

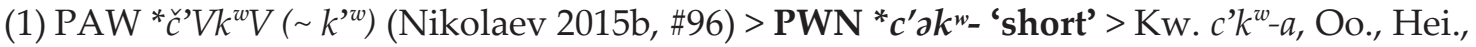
Hai. $c^{\prime} k^{w} \bullet$ PAlg *tatkw-, * ${ }^{*} a c ̌ k w-,{ }^{*} t e t k w-$ 'short' > Yu. $t k^{w-}$ 'to be short'; PA *tatk-, *tahk-, *čahk-> WAb. takkw-; FSCr. čahkw- 'short'.

(2) PWS *n'i:c-> Noo. $n^{\prime} i: c$ 'short' (also Mak. di:c'-a(q)).

(3) PNi ${ }^{*} p h \chi-a q->$ NiA. $p(h) \chi$-aq-, NiS. ph $\chi$-aq-t 'short'.

(4) MiPe. ehkw- 'short'.

106. SNAKE

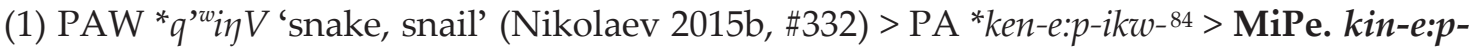
$i k w$ - $a$, FSCr. kin-ep-ik'snake'.

(2) PWN *si:t- 'snake' > Kw., Oo. sit-mo, Hei. sít-m.

(3) $\mathrm{PW} * \chi i$ :- 'to crawl' > Noo. hi:-yi 'snake'.

(4) $\mathrm{PNi}$ *uml- > NiA. uml-ak, NiS. uml-ay(a) 'snake'.

(5) PA *atko:k- 'snake' > WAb. sskkok 'snake, worm'. See WORM.

(6) Wi. Pa?roč 'snake'.

(7) Yu le?y-es, ley-es 'snake'.

107. THIN ${ }^{85}$

(1) PNi *nok-> NiA., NiS. nok-la- 'thin, narrow'.

(2) PA * pi:w- 'small piece' > WAb. piw-i ‘small, fine, thin'. See SMALL.

(3) PA * mya:l- 'bad' > MiPe. mya:l- 'thin'.

(4) PA *pap-ak-, *pep-ak- 'thin, lean' > FSCr. papak- 'thin'.

(5) Noo. ?anik-it 'thin' (cf. 2anik-s 'in length').

(6) Yu. mes- 'thin, narrow, slim'.

108. WIND ${ }^{86}$

(1) PAW *layVwV 'wind; to blow (wind)' (cf. Nikolaev 2015b, \#192) > PW *yu:- 'wind' > PWN *yu:- 'wind, draft' > Kw. yo-la, Oo. yu-ala, Hei. yu-ála, Hai. yu-ala; PWS *yu(:)- > Noo. $y u-2 i$ 'wind blowing' (also Dit. yu- id., Mak. yuyu:-qsi:s 'North Wind') • PNi *la > NiA., NiS. la 'wind' • PAlg *lo(:)yew-, *ro(:)yew- 'wind, to blow' ${ }^{87}>$ Yu. ro:- $\boldsymbol{k}^{w}$ 'wind'.

(2) PA *ata:m-, *ałam- 'breath' > WAb. alóm-ss-ekk 'wind', MiPe. ala:m-ihs-en- II 'it is windy'.

${ }^{83}$ No data for Wiyot.

${ }^{84} \mathrm{PA}$ *kenw-e:p-ikw- 'snake' has -w- by analogy with *kenw- 'long'.

${ }^{85}$ No data for PWN and Wiyot.

${ }^{86}$ No data for FSCr. and Wiyot.

${ }^{87}$ Also PA *lo:w- 'to blow (wind)'. 
109. WORM ${ }^{88}$

(1) PAW * $x^{w} O: \eta V \sim{ }^{*} \eta O: x^{w} V$ 'bee’ (Nikolaev 2015b, \#393a) > PA *(a:)mo:-hs- > MiPe. $m u$ :-hs-i-a 'insect, worm, bug'.

(2) PAW * $k^{\text {ww }} \dot{i} l V \eta V\left(\sim\right.$ ) 'worm' (Nikolaev 2015b, \#185) > Yu. Pyek ${ }^{w t}$ 'maggot, worm'.

(3) $\mathrm{PNi}$ *chxev-r > NiA. chxev-r, NiS. chxev-ř 'worm'.

(4) PA *ałko:k- 'snake' > WAb. sskkok 'snake, worm'. See SNAKE.

(3) Noo. titk-'umc 'any worm, including the mussel worm (Nereis vexillosa)' (cf. titk 'to jerk, pull fast').

110. YEAR ${ }^{89}$

(1) PAW *2äń $V$ 'year, season' (Nikolaev 2015b, \#10) > PNi *ań > NiA., NiS. ań 'year'.

(2) PA *li:k- 'to grow; give birth; loosen' > WAb. lik-at-en 'it is a year'.

(3) Noo. -qPičh 'year'.

(4) Yu. lok (loks-) 'to be a year'.

(5) MiPe. kihkatwi 'year'.

\section{Language abbreviations and sources}

Dit. - Ditidaht (Nitinaht), acc. to Fortescue 2007.

FSCr. - Fort Severn Cree, acc. to MacKenzie 2005.

Hai. - Haisla, acc. to Linkoln, Rath 1980 and Fortescue 2007..

Hei. - Heiltsuk, acc. to Linkoln, Rath 1980.

Kw. - Kwak'wala (Kwakiutl), acc. to Linkoln, Rath 1980.

Mak. - Makah, acc. to Fortescue 2007.

Men. - Menominee, acc. to Hewson 1993 and Oleg Mudrak's comparative Proto-Algonquian database (ms.).

MiPe. - Miami-Peoria, acc. to Baldwin, Costa 2005.

Mic. - Micmac (Mi'kmaq), acc. to Oleg Mudrak’s comparative Proto-Algonquian database (ms.).

NiA. - Amur Nivkh, acc. to materials in Oleg Mudrak's comparative Nivkh database nivget.dbf (ms.).

NiS. - Sakhalin Nivkh, acc. to materials in O. Mudrak's comparative Nivkh database nivget.dbf (ms.).

Noo. - Nootka (Nuuchahnulth), acc. to Stonham 2005.

Oo. - Oowekyala, acc. to Linkoln, Rath 1980.

PA - Proto-Algonquian, acc. to Aubin 1975; Goddard 1974, 1979, 1982; Hewson 1993; Proulx 1984a, b, 1989, 1991, 1992, $1994^{90}$.

PAlg - Proto-Algic, acc. to Proulx 1984a, b, 1991, 1992, $1994^{91}$

PAW - Proto-Algonquian-Wakashan, acc. to S. Nikolaev's reconstruction.

PNA - Proto-Nivkh-Algic, acc. to S. Nikolaev's reconstruction.

PNi - Proto-Nivkh, acc. to S. Nikolaev's reconstruction and based on materials in Oleg Mudrak's comparative Nivkh database nivget. dbf (ms.).

PW - Proto-Wakashan, acc. to Fortescue $2007^{92}$.

PWN - Proto-Southern Wakashan, acc. to Fortescue 2007.

PWS - Proto-Northern Wakashan, acc. to Fortescue 2007 and Linkoln, Rath 1980.

${ }^{88}$ No data for PWN, FSCr., or Wiyot.

${ }^{89}$ No data for PWN, FSCr., or Wiyot.

${ }^{90}$ With the following elements of transliteration: ${ }^{*} \vartheta \Rightarrow{ }^{*} t ;{ }^{*} x k,{ }^{*} x p \Rightarrow{ }^{*} t k,{ }^{*} t p ;{ }^{*} c ̧ k,{ }^{*} c ̧ p>{ }^{*} s k,{ }^{*} s p$.

${ }^{91}$ Several PAlg roots have also been added by myself in accordance with Paul Proulx's rules of reconstruction. I interpret the PAlg phonemes that Proulx denotes as ${ }^{*} \mathrm{~T},{ }^{*} \mathrm{~K},{ }^{*} \mathrm{~L},{ }^{*} \mathrm{C},{ }^{*} \mathrm{C}$ as voiced consonants $\left({ }^{*} d,{ }^{*} g,{ }^{*} L,{ }^{*} 3,{ }^{*}\right)$. Proulx's *S which only occurs in clusters is reinterpreted by myself as the voiced fricative * $\gamma$; it is reflected as PA *?, Wi. $\varnothing$ and Yu. $s\left(<^{*} r<{ }^{*} \gamma\right)$. This PAlg phoneme corresponds to velars in other Algonquian-Wakashan languages. The "normal" PAlg velar glide * $\gamma$ does not occur in consonantal clusters.

${ }^{92}$ I have added several PW roots according to M. Fortescue's rules of reconstruction. The same applies to PWN and PWS. 
Quil. - Quileute, acc. to Powell, Woodruff 1976.

WAb. - Western Abenaki, acc. to Day 1995.

Wi. - Wiyot, acc. to Teeter, Nichols 1993 and Proulx 1984a, b, 1989, 1991, 1992, 1994.

Yu. - Yurok, acc. to Robins 1958 and Proulx 1985.

\section{References}

Aubin, George F. 1975. A Proto-Algonquian dictionary. Ottawa: National Museums Of Canada.

Baldwin, Daryl; David J. Costa. 2005. A Miami-Peoria dictionary. Miami: Myaamia Publications.

Day, Gordon M. 1995. Western Abenaki dictionary. Vols. 1-2. Hull: Canadian Museum of Civilization.

Fortescue, Michael. 2007. Comparative Wakashan dictionary. München: LINCOM Europa.

Hewson, John. 1993. A computer-generated dictionary of Proto-Algonquian. Hull: Canadian Museum of Civilization.

Lincoln, Neville J., John C. Rath. 1980. North Wakashan Comparative Root List. Canadian Museum of Civilization Mercury Series, Canadian Ethnology Service Paper no. 68. Ottawa: National Museums of Canada.

MacKenzie, Marguerite. 2005. Wasaho Ininîmowin dictionary (Fort Severn Cree). Cree-English / English-Cree. A draft. Canada.

Nikolaev, Sergei L. 2015a. Toward the reconstruction of Proto-Algonquian-Wakashan. Part 1: Proof of the Algonquian-Wakashan relationship. Journal of Language Relationship 13(1): 23-61.

Nikolaev, Sergei L. 2015b. Toward the Reconstruction of Proto-Algonquian-Wakashan. Part 2: Algonquian-Wakashan Sound Correspondences // Journal of Language Relationship 13(4): 289-328.

Powell, J.V., Fred Woodruff, Sr. 1976. Quileute dictionary. Northwest anthropological research notes: Memoir No. 3 , Part 2. Moscow: University of Idaho.

Proulx, Paul. 1984a. Proto-Algic II: Verbs. International Journal Of American Linguistics 50(1): 59-94.

Proulx, Paul. 1984b. Proto-Algic I: Phonological sketch. International Journal Of American Linguistics 50(2): 165-207.

Proulx, Paul. 1989. A sketch of Blackfoot historical phonology. International Journal Of American Linguistics 55(1): 43-82.

Proulx, Paul. 1991. Proto-Algic III: Pronouns. Kansas Working Papers in Linguistics 16: 129-170.

Proulx, Paul. 1992. Proto-Algic IV: Nouns. Kansas Working Wapers in Linguistics 17(2): 11-58.

Proulx, Paul. 1994. Proto-Algic V: Doublets and their implications. Kansas Working Papers in Linguistics 19(2): 113-183.

Robins, Robert H. 1958. The Yurok Language: Grammar, Texts, Lexicon. University of California Press.

Stonham, John. 2005. A concise dictionary of the Nuuchahnulth language of Vancouver Island. Lewiston-QueenstonLampeter: Edwin Mellen Press.

Teeter, Karl V., John D. Nichols. 1993. Wiyot Handbook I. Glossary and concordance. Winnipeg: Algonquian and Iroquoian Linguistics.

С. А. Николаев. К реконструкции алгонкино-вакашского праязыка. Ч. 3: Алгонкиновакашский 110-словный список.

Третья часть комплексного исследования, посвященного обоснованию алгонкиновакашской семьи языков, в которую, помимо нескольких языковых групп Северной Америки, также включается нивхский язык, представляет собой аннотированную демонстрацию сравнительно-исторических данных, использованных при лексикостатистических подсчетах между языками этой гипотетической семьи. Поскольку лексикостатистический материал нуждается в подробном историческом комментарии, из соображений объема он не мог быть включен в предыдущие две части исследования. Помимо этого, в данной части исследования также приведены реконструкции для ряда новых праалгонкино-вакашских и пранивхско-алгонкинских корней. В лексикостатистических подсчетах задействованы следующие языки: прасеверновакашский (примерная глоттохронологическая дата распада - ок. 800 г. н. э.) и записанные в XIX-XX вв. языки нутка (нучахнулт), нивхский (амурский и сахалинский диалекты), западный абенаки, майами-пеория, кри из Форт Северна, вийот и юрок.

Ключевые слова: алгонкино-вакашсике языки, нивхско-алгонкинские языки, алгические языки, вакашские языки, чимакум-вакашские языки, нивхский язык, историческая фонетика, сравнительный словарь, лексикостатистика. 